\title{
GUARANTEED LOWER BOUNDS FOR EIGENVALUES
}

\author{
CARSTEN CARSTENSEN AND JOSCHA GEDICKE
}

\begin{abstract}
This paper introduces fully computable two-sided bounds on the eigenvalues of the Laplace operator on arbitrarily coarse meshes based on some approximation of the corresponding eigenfunction in the nonconforming Crouzeix-Raviart finite element space plus some postprocessing. The efficiency of the guaranteed error bounds involves the global mesh-size and is proven for the large class of graded meshes. Numerical examples demonstrate the reliability of the guaranteed error control even with an inexact solve of the algebraic eigenvalue problem. This motivates an adaptive algorithm which monitors the discretisation error, the maximal mesh-size, and the algebraic eigenvalue error. The accuracy of the guaranteed eigenvalue bounds is surprisingly high with efficiency indices as small as 1.4 .
\end{abstract}

\section{INTRODUCTION}

The well-established Rayleigh-Ritz principle for the algebraic as well as for the continuous eigenvalues of the Laplacian,

$$
-\Delta u=\lambda u \quad \text { for } u \in V \backslash\{0\}:=H_{0}^{1}(\Omega) \backslash\{0\},
$$

immediately results in upper bounds of the eigenvalues by Rayleigh quotients

$$
\lambda_{1} \leq R(v):=\|v\|^{2} /\|v\|^{2} \quad \text { for any } v \in V \backslash\{0\} .
$$

Standard notation on Lebesgue and Sobolev spaces and norms is adopted throughout this paper and, for brevity, $\|\cdot\|:=\|\cdot\|_{L^{2}(\Omega)}$ denotes the $L^{2}$ norm and $\|\cdot\|:=$ $\|\nabla \cdot\|_{L^{2}(\Omega)}:=|\cdot|_{H^{1}(\Omega)}$ denote the $H^{1}$ semi-norm for the entire bounded polygonal Lipschitz domain $\Omega$. Although $\lambda_{1}$ in (1.2) denotes the first exact eigenvalue of (1.1), the well-established min-max principle applies to the higher eigenvalues $0<\lambda_{1}<\lambda_{2} \leq \lambda_{3} \leq \ldots$. Since upper bounds are easily obtained by conforming discretisations via (1.2), the computation of lower bounds is of high interest and we solely mention the milestones [For55, Wei56, AD04] for asymptotic lower bounds in the sense that they provide guaranteed bounds under the assumption that the global mesh-size is sufficiently small. Unfortunately, the minimal mesh-size required to deduce some guaranteed lower eigenvalue bound is not quantified in the current literature - so nobody knows whether some mesh allows some guaranteed bound or not. This paper establishes guaranteed lower bounds even for very coarse triangulations like those of Figure 1.1 for the unit square $\Omega=(0,1)^{2}$ with only two

Received by the editor January 11, 2012 and, in revised form, October 28, 2012 and March 12, 2013.

2010 Mathematics Subject Classification. Primary 65N15, 65N25, 65N30.

Key words and phrases. Eigenvalue, adaptive finite element method, bounds.

This paper was supported by the DFG Research Center MATHEON "Mathematics for key technologies", and the graduate school BMS "Berlin Mathematical School" in Berlin, and the World Class University (WCU) program through the National Research Foundation of Korea (NRF) funded by the Ministry of Education, Science and Technology R31-2008-000-10049-0.

(C) 2014 American Mathematical Society 2605

Reverts to public domain 28 years from publication 

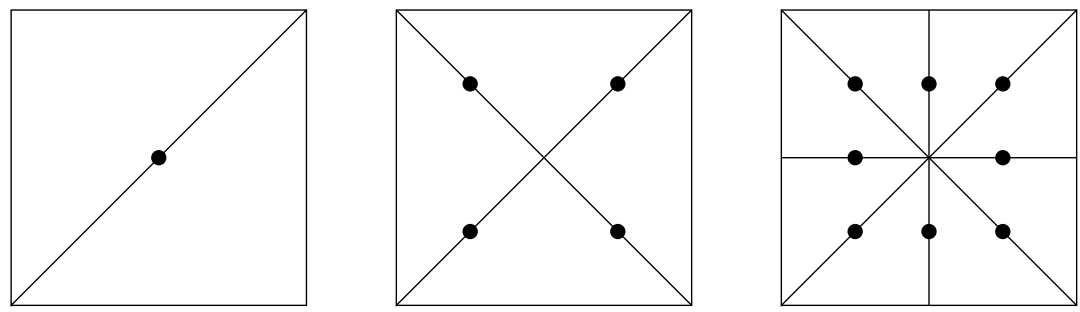

Figure 1.1. Criss (left), criss-cross (middle) and union-jack (right) triangulations of the unit square in 2, 4, and 8 congruent triangles.

triangles. For the three meshes of Figure 1.1 clearly in the pre-asymptotic range of convergence, Theorem 3.1 of this paper provides the guaranteed bounds

$$
2.3371 \leq \lambda_{1} \leq 32, \quad 4.2594 \leq \lambda_{1} \leq 24, \quad \text { and } \quad 6.6182 \leq \lambda_{1} \leq 22.0397
$$

for the first exact eigenvalue $\lambda_{1}=2 \pi^{2}=19.7392$ despite the coarse discretisation with just 1, 4, or 8 degrees of freedom in a Crouzeix-Raviart nonconforming finite element discretisation (CR-NCFEM); cf. Examples 3.7 and 3.10 below for more details.

To the best knowledge of the authors, any other a posteriori error control requires some (unquantified) sufficiently small global mesh-size [CG11, DDP12, DPR03]; for an a priori error analysis see BO91, SF73. The asymptotic convergence of the conforming FEM is presented in [CG11, GMZ09, GG09] and the asymptotic quasi-optimal convergence and complexity in CG12b, DRSZ08, DXZ08. Recently, HHL11 proves asymptotic lower bounds of several nonconforming FEM and higher order elliptic operators. The main results of this paper are by no means restricted to the present case and work for 3D as well as for biharmonic eigenvalue problems CG12a.

To describe the main results of this paper, let $\mathcal{T}$ be an arbitrarily coarse shaperegular triangulation of the polygonal domain $\Omega$ into triangles with set $\mathcal{E}$ of edges and let

$$
C R_{0}^{1}(\mathcal{T}):=\left\{v \in P_{1}(\mathcal{T}) \mid v \text { is continuous at } \operatorname{mid}(\mathcal{E}) \text { and } v=0 \text { at } \operatorname{mid}(\mathcal{E}(\partial \Omega))\right\}
$$

denote the Crouzeix-Raviart nonconforming FEM spaces for the piecewise firstorder polynomials $P_{1}(\mathcal{T}):=\left\{v \in L^{2}(\Omega)|\forall T \in \mathcal{T}, v|_{T}\right.$ is affine $\}$. The degrees of freedom for a triangle are depicted in Figure 1.2. Suppose that $\left(\tilde{\lambda}_{C R, 1}, \tilde{u}_{C R, 1}\right) \in$ $\mathbb{R} \times C R_{0}^{1}(\mathcal{T})$ is some computed approximation of the smallest exact eigenvalue $\lambda_{1}$ of the associated algebraic eigenvalue problem with the stiffness matrix $\mathbf{A}$, the (diagonal) mass matrix $\mathbf{B}$, and the algebraic residual $\mathbf{r}:=\mathbf{A} \tilde{\mathbf{u}}_{C R, 1}-\tilde{\lambda}_{C R, 1} \mathbf{B} \tilde{\mathbf{u}}_{C R, 1}$ for the algebraic eigenvector $\tilde{\mathbf{u}}_{C R, 1}$. Suppose that the first approximated discrete eigenvalue $\tilde{\lambda}_{C R, 1}$ is closer to the first discrete eigenvalue $\lambda_{C R, 1}$ than to the second discrete eigenvalue (which has to be guaranteed by algebraic eigenvalue analysis) and that $\|\mathbf{r}\|_{\mathbf{B}^{-1}}<\tilde{\lambda}_{C R, 1}$. The numerical experiments of Section 6 show that for the simple first eigenvalue the algebraic separation condition is not critical, but a cluster of eigenvalues may lead to difficulties with this separation condition on the level of the algebraic eigensolve. The first main result, in Theorem 3.1 below, 


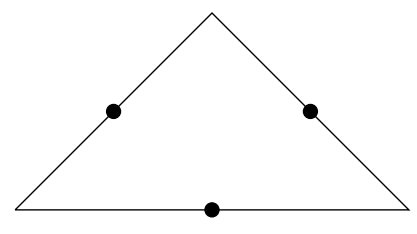

Figure 1.2. Crouzeix-Raviart finite element.

implies

$$
\frac{\tilde{\lambda}_{C R, 1}-\|\mathbf{r}\|_{\mathbf{B}^{-1}}}{1+\kappa^{2}\left(\tilde{\lambda}_{C R, 1}-\|\mathbf{r}\|_{\mathbf{B}^{-1}}\right) H^{2}} \leq \lambda_{1} \leq R\left(\mathcal{I}_{C M} \tilde{u}_{C R, 1}\right) .
$$

Since $\tilde{\lambda}_{C R, 1}$ is the nearest approximation to $\lambda_{C R, 1}$, the algebraic residual $\mathbf{r}$ yields an upper bound for the discrete eigenvalue error in Lemma 3.8. Moreover, $H:=$ $\max _{T \in \mathcal{T}} \operatorname{diam}(T)$ denotes the maximal mesh-size and $\mathcal{I}_{C M}$ denotes the interpolation operator of Section 3 which ensures $\mathcal{I}_{C M} \tilde{u}_{C R, 1} \not \equiv 0$ to define the Rayleigh quotient. The explicit constant $\kappa$ reads $\kappa^{2}:=\left(1 / 8+j_{1,1}^{-2}\right) \leq 0.1932$ for the first positive root $j_{1,1}$ of the Bessel function of the first kind.

Note that the nonconforming eigenvalue for the first two meshes of Figure 1.1 reads $\lambda_{C R, 1}=24$ and is larger than the solution $\lambda=2 \pi^{2}$. This novel observation shows that the nonconforming eigenvalue by itself does not always provide some lower bound for arbitrarily coarse meshes in contrast to the lower bound given in this paper. The asymptotic a posteriori error control of AD04 does not provide those error bounds.

The second main result, Theorem 4.1, guarantees efficiency in the sense that the difference of the upper and lower bound is bounded by the error for the large class of graded meshes.

The lower bound is generalised to higher eigenvalues under some explicit given mesh-size restriction plus the aforementioned separation condition. Together with a conforming approximation for an upper bound, the bounds for the higher eigenvalues are also efficient.

The efficiency for graded meshes motivates the development of an adaptive algorithm that balances the finite element error and the global mesh-size $H$ in order to reduce the difference of the upper and lower eigenvalue bounds.

The remaining parts of this paper are organised as follows. Section 2 presents the model problem (1.1) and the necessary notation. Section 3 proves the explicit lower and upper bounds for the smallest eigenvalue based on the nonconforming discrete eigenvalue as well as on its approximation. The efficiency of the resulting a posteriori error estimator follows in Section 4. Section 5 establishes some bounds for higher eigenvalues and their efficiency. Section 6 presents some adaptive algorithm which monitors the discretisation error, the maximal mesh-size, and the algebraic eigenvalue error and verifies the theoretical results in some numerical experiments. An empirical comparison of conforming and nonconforming discretisations is included as well. Since the consistent mass matrix is diagonal, nonconforming discretisations are of particular attraction in practise.

Throughout this paper, $A \lesssim B$ abbreviates the inequality $A \leq C B$ for some constant $C$ that does not depend on the mesh-sizes but only on some lower bound of the minimal interior angle in $\mathcal{T}$. 


\section{Notation And Preliminaries}

The weak formulation of the model problem (1.1) looks for the eigenpair $(\lambda, u) \in$ $\mathbb{R} \times V$ with $b(u, u)=1$ and

$$
a(u, v)=\lambda b(u, v) \quad \text { for all } v \in V:=H_{0}^{1}(\Omega) .
$$

Here and throughout this paper, the scalar products $a(\cdot, \cdot)$ and $b(\cdot, \cdot)$ read

$$
a(u, v):=\int_{\Omega} \nabla u \cdot \nabla v d x \quad \text { and } \quad b(u, v):=\int_{\Omega} u v d x \quad \text { for all } u, v \in V
$$

and induce the norms $\|\cdot\|:=a(\cdot, \cdot)^{1 / 2}$ on $\mathrm{V}$ and $\|\cdot\|:=b(\cdot, \cdot)^{1 / 2}=\|\cdot\|_{L^{2}(\Omega)}$ on $L^{2}(\Omega)$.

The eigenvalue problem is symmetric and positive definite and there exist countably many positive eigenvalues with no finite accumulation point [BO91]. The eigenvalues can be ordered

$$
0<\lambda_{1}<\lambda_{2} \leq \lambda_{3} \leq \ldots
$$

and there exist some orthonormal basis $\left(u_{1}, u_{2}, u_{3}, \ldots\right)$ of corresponding eigenvectors. Section 3 focuses on the computation of the first eigenvalue $\lambda_{1}$ which is simple [Eva00, Section 6.5, Theorem 2]. The min-max principle reduces for the smallest eigenvalue to

$$
\lambda_{1}=\min _{v \in V \backslash\{0\}} R(v) \quad \text { with the Rayleigh quotient } \quad R(v):=a(v, v) / b(v, v) .
$$

Let $\mathcal{T}$ be a regular triangulation in the sense of Ciarlet of the bounded 2D Lipschitz domain $\Omega$ into at least two triangles such that all $T \in \mathcal{T}$ are closed triangles with positive area $|T|$ and two distinct intersecting triangles $T_{1}, T_{2} \in \mathcal{T}$ share either one common edge or one common node. Let $\mathcal{E}$ denote the set of all edges $(\mathcal{E}(\Omega)$ of interior edges) of the triangulation $\mathcal{T}$, let $\operatorname{mid}(E)$ be the midpoint and $h_{E}$ the length of an edge $E \in \mathcal{E}$. Let $h_{T}:=\operatorname{diam}(T), H:=\max _{T \in \mathcal{T}} h_{T}$ and $h_{\mathcal{T}} \in P_{0}(\mathcal{T})$ piecewise defined as $\left.h_{\mathcal{T}}\right|_{T}=h_{T}$. Let $[\cdot]_{E}:=\left.(\cdot)\right|_{T_{+}}-\left.(\cdot)\right|_{T_{-}}$denote the jump across an interior edge $E \in \mathcal{E}(\Omega)$ with $E=T_{+} \cap T_{-}, T_{ \pm} \in \mathcal{T}$, and $[\cdot]_{E}:=(\cdot)$ for $E \subset \partial \Omega$. Let $\mathcal{N}$ denote the set of all nodes $(\mathcal{N}(\Omega)$ of interior nodes) in the triangulation $\mathcal{T}$.

The conforming finite element space is defined by $V_{C}(\mathcal{T}):=H_{0}^{1}(\Omega) \cap P_{1}(\mathcal{T})$. In the following let $\Pi_{0}$ denote the $L^{2}$ projection onto piecewise constants $P_{0}(\mathcal{T})$ as well as $P_{0}\left(\mathcal{T} ; \mathbb{R}^{n}\right)$.

For all interior edges $E \in \mathcal{E}(\Omega)$, the edge-oriented basis function $\psi_{E}$ is defined by

$$
\psi_{E}(\operatorname{mid}(E))=1 \quad \text { and } \quad \psi_{E}(\operatorname{mid}(F))=0 \text { for all } F \in \mathcal{E} \backslash E .
$$

Then $C R_{0}^{1}(\mathcal{T})=\operatorname{span}\left\{\psi_{E} \mid E \in \mathcal{E}(\Omega)\right\} \nsubseteq V$ and the nonconforming discrete eigenvalue problem reads:

Find an eigenpair $\left(\lambda_{C R}, u_{C R}\right) \in \mathbb{R} \times C R_{0}^{1}(\mathcal{T})$ with $b\left(u_{C R}, u_{C R}\right)=1$ and

$$
a_{N C}\left(u_{C R}, v_{C R}\right)=\lambda_{C R} b\left(u_{C R}, v_{C R}\right) \quad \text { for all } v_{C R} \in C R_{0}^{1}(\mathcal{T}) \text {. }
$$

The nonconforming bilinear form $a_{N C}$,

$$
a_{N C}\left(u_{C R}, v_{C R}\right):=\sum_{T \in \mathcal{T}} \int_{T} \nabla u_{C R} \cdot \nabla v_{C R} d x \quad \text { for all } u_{C R}, v_{C R} \in C R_{0}^{1}(\mathcal{T})
$$


induces the mesh-dependent norm $\|\cdot\|_{N C}:=a_{N C}(\cdot, \cdot)^{1 / 2}$ and the Rayleigh quotient

$$
R_{N C}\left(v_{C R}\right):=a_{N C}\left(v_{C R}, v_{C R}\right) /\left\|v_{C R}\right\|^{2} \quad \text { for all } v_{C R} \in C R_{0}^{1}(\mathcal{T}) \backslash\{0\} .
$$

The nonconforming interpolant $\mathcal{I}_{N C}: V \rightarrow C R_{0}^{1}(\mathcal{T})$ is defined for any $v \in V$ by

$$
\mathcal{I}_{N C} v(\operatorname{mid}(E)):=\frac{1}{|E|} \int_{E} v d s \text { for all } E \in \mathcal{E} \text {. }
$$

The proof of the $L^{2}$ error estimate below is essentially contained in CGR12.

Theorem 2.1 ( $L^{2}$ interpolation error estimate). Any $v \in H_{0}^{1}(\Omega)$ satisfies

$$
\left\|v-\mathcal{I}_{N C} v\right\| \leq \kappa H\left\|v-\mathcal{I}_{N C} v\right\|_{N C} .
$$

Proof. The proof reduces to the corresponding estimate on a single triangle $T$. Let $f \in H^{1}(T)$ satisfy $\int_{E} f \mathrm{ds}=0$ on the triangle $T=\operatorname{conv}(\{P\} \cup E)$ with an edge $E$ opposite to the vertex $P$. Then Lemma 2.2 in CGR12 proves for the first positive root $j_{1,1}$ of the Bessel function $J_{1}$ of the first kind that the following holds:

$$
\|f\|_{L^{2}(T)} \leq \sqrt{\max _{x \in E}|P-x|^{2} / 8+h_{T}^{2} / j_{1,1}^{2}}|f|_{H^{1}(T)} .
$$

The choice $f:=v-\mathcal{I}_{N C} v$ concludes the proof.

\section{EXPLICIT BOUNDS FOR THE SMALLEST EIGENVALUE}

This section is devoted to the proof of the explicit bounds for the first eigenvalue $\lambda_{1}$. Recall that $H$ is the maximal diameter in the triangulation $\mathcal{T}$ and that $\kappa$ is some universal constant.

Theorem 3.1. Let $\left(\tilde{\lambda}_{C R, 1}, \tilde{u}_{C R, 1}\right) \in \mathbb{R} \times C R_{0}^{1}(\mathcal{T})$ be an approximation of the eigenpair $\left(\lambda_{1}, u_{1}\right)$ of the smallest eigenvalue with $\left\|\tilde{u}_{C R, 1}\right\|_{L^{2}(\Omega)}=1$ and with algebraic residual $\mathbf{r}:=\mathbf{A} \tilde{\mathbf{u}}_{C R, 1}-\tilde{\lambda}_{C R, 1} \mathbf{B} \tilde{\mathbf{u}}_{C R, 1}$ and let $\mathcal{I}_{C M} \tilde{u}_{C R, 1}$ be the quasi-interpolant of $\tilde{u}_{C R, 1}$ from Definition 3.3 below. Suppose separation of $\tilde{\lambda}_{C R, 1}$ from the remaining discrete spectrum in the sense that $\tilde{\lambda}_{C R, 1}$ is closer to the smallest discrete eigenvalue $\lambda_{C R, 1}$ than to any other discrete eigenvalue and suppose that $\|\mathbf{r}\|_{\mathbf{B}^{-1}}<\tilde{\lambda}_{C R, 1}$. Then it holds that

$$
\frac{\tilde{\lambda}_{C R, 1}-\|\mathbf{r}\|_{\mathbf{B}^{-1}}}{1+\kappa^{2}\left(\tilde{\lambda}_{C R, 1}-\|\mathbf{r}\|_{\mathbf{B}^{-1}}\right) H^{2}} \leq \lambda_{1} \leq R\left(\mathcal{I}_{C M} \tilde{u}_{C R, 1}\right) .
$$

The remaining part of this section is devoted to the proof of Theorem 3.1. The point of departure is the particular case of exact solve.

Theorem 3.2 (Lower bound for exact solve). The first exact eigenvalue $\lambda_{1}$ and the first discrete eigenvalue $\lambda_{C R, 1}$ satisfy

$$
\frac{\lambda_{C R, 1}}{1+\kappa^{2} \lambda_{C R, 1} H^{2}} \leq \lambda_{1}
$$

Proof. The Pythagoras theorem in $L^{2}\left(\Omega ; \mathbb{R}^{2}\right)$ reads

$$
\lambda_{1}=a\left(u_{1}, u_{1}\right)=\left\|\nabla u_{1}\right\|^{2}=\left\|\nabla u_{1}-\Pi_{0} \nabla u_{1}\right\|^{2}+\left\|\Pi_{0} \nabla u_{1}\right\|^{2} .
$$


An integration by parts on one triangle $T \in \mathcal{T}$ and $\int_{E}\left(v-\mathcal{I}_{N C} v\right) d s=0$, for all $v \in V$ and $E \in \mathcal{E}$, show that

$$
\begin{aligned}
\left.|T| \Pi_{0} \nabla v\right|_{T} & =\int_{T} \nabla v d x=\int_{\partial T} v \nu_{T} d s \\
& =\int_{\partial T}\left(\mathcal{I}_{N C} v\right) \nu_{T} d s=\int_{T} \nabla\left(\mathcal{I}_{N C} v\right) d x=|T| \nabla\left(\left.\mathcal{I}_{N C} v\right|_{T}\right) .
\end{aligned}
$$

This proves the known identity for the piecewise defined gradient $\left.\left(\nabla_{N C} \cdot\right)\right|_{T}:=$ $\nabla\left(\left.\cdot\right|_{T}\right):$

$$
\Pi_{0} \nabla v=\nabla_{N C}\left(\mathcal{I}_{N C} v\right) .
$$

The combination with the aforementioned Pythagoras identity reads

$$
\lambda_{1}=\left\|u_{1}-\mathcal{I}_{N C} u_{1}\right\|_{N C}^{2}+\left\|\mathcal{I}_{N C} u_{1}\right\|_{N C}^{2} .
$$

The min-max principle on the discrete eigenvalue problem allows the estimate

$$
\lambda_{C R, 1}\left\|\mathcal{I}_{N C} u_{1}\right\|^{2} \leq\left\|\mathcal{I}_{N C} u_{1}\right\|_{N C}^{2} .
$$

The combination of the previous results leads to

$$
\left\|u_{1}-\mathcal{I}_{N C} u_{1}\right\|_{N C}^{2}+\lambda_{C R, 1}\left\|\mathcal{I}_{N C} u_{1}\right\|^{2} \leq \lambda_{1} .
$$

Some elementary algebra based on $\left\|u_{1}\right\|=1$ and the binomial expansion yield

$$
\begin{aligned}
1+\left\|u_{1}-\mathcal{I}_{N C} u_{1}\right\|^{2} & -2\left\|u_{1}-\mathcal{I}_{N C} u_{1}\right\| \\
& \leq 1+\left\|u_{1}-\mathcal{I}_{N C} u_{1}\right\|^{2}-2 b\left(u_{1}-\mathcal{I}_{N C} u_{1}, u_{1}\right)=\left\|\mathcal{I}_{N C} u_{1}\right\|^{2} .
\end{aligned}
$$

Set $s=\alpha /(1+\alpha)$ with $\alpha:=\kappa^{2} H^{2} \lambda_{C R, 1}$. This results in

$$
1+\left\|u_{1}-\mathcal{I}_{N C} u_{1}\right\|^{2}-2 s\left\|u_{1}-\mathcal{I}_{N C} u_{1}\right\|-2(1-s)\left\|u_{1}-\mathcal{I}_{N C} u_{1}\right\| \leq\left\|\mathcal{I}_{N C} u_{1}\right\|^{2} .
$$

The Young inequality $2 s\left\|u_{1}-\mathcal{I}_{N C} u_{1}\right\| \leq s^{2}+\left\|u_{1}-\mathcal{I}_{N C} u_{1}\right\|^{2}$ leads to

$$
1-s^{2}-2(1-s)\left\|u_{1}-\mathcal{I}_{N C} u_{1}\right\| \leq\left\|\mathcal{I}_{N C} u_{1}\right\|^{2} .
$$

The a priori estimate of Theorem 2.1] plus another Young inequality

$$
2\left\|u_{1}-\mathcal{I}_{N C} u_{1}\right\|_{N C} \leq t+\left\|u_{1}-\mathcal{I}_{N C} u_{1}\right\|_{N C}^{2} / t
$$

for $t:=(1-s) \kappa H \lambda_{C R, 1}>0$ result in

$$
1-s^{2}-(1-s)^{2} H^{2} \kappa^{2} \lambda_{C R, 1}-\left\|u_{1}-\mathcal{I}_{N C} u_{1}\right\|_{N C}^{2} / \lambda_{C R, 1} \leq\left\|\mathcal{I}_{N C} u_{1}\right\|^{2} .
$$

The combination of (3.2) and (3.3) proves

$$
\lambda_{C R, 1}\left(\left(1-s^{2}\right)-((1-s) \kappa H)^{2} \lambda_{C R, 1}\right) \leq \lambda_{1} .
$$

This and the definition of $s$ lead to

$$
\frac{\lambda_{C R, 1}}{1+\kappa^{2} H^{2} \lambda_{C R, 1}} \leq \lambda_{1}
$$

For the analysis of an upper bound, notice that the min-max principle for the smallest eigenvalue shows

$$
\lambda_{1}=\min _{v \in V \backslash\{0\}} R(v) \leq R(w) \text { for any } w \in V_{C}(\mathcal{T}) \backslash\{0\} .
$$

Thus, any conforming approximation close to the nonconforming eigenfunction provides a guaranteed upper bound. The postprocessing of CM13 provides such a sufficiently accurate conforming interpolation $\mathcal{I}_{C M}: C R_{0}^{1}(\mathcal{T}) \rightarrow V_{C}\left(\mathcal{T}^{*}\right)$ for the 


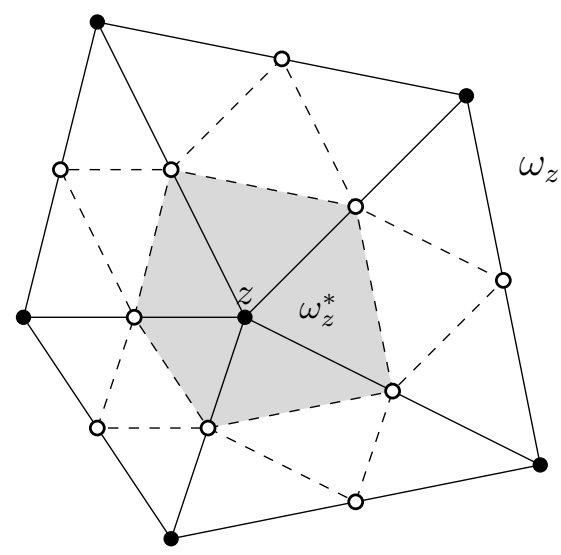

Figure 3.1. Patch $\omega_{z}$ of $\mathcal{T}$ (solid lines) with refined patch $\omega_{z}^{*}$ (grey) of the sub-triangulation $\mathcal{T}^{*}:=\operatorname{red}(\mathcal{T})$ (solid and dashed lines).

red-refined triangulation $\mathcal{T}^{*}:=\operatorname{red}(\mathcal{T})$ of $\mathcal{T}$ into triangles depicted in Figure 3.1 (The red-refined triangulation $\operatorname{red}(\mathcal{T})$ results from dividing each triangle in $\mathcal{T}$ into 4 congruent sub-triangles by connecting the midpoints of the edges by straight lines.)

Definition 3.3. For all nodes $z$ in the red-refined triangulation $\mathcal{T}^{*}=\operatorname{red}(\mathcal{T})$ and $v_{C R} \in C R_{0}^{1}(\mathcal{T})$, set

$$
\mathcal{I}_{C M} v_{C R}(z):= \begin{cases}0 & \text { if } z \text { lies on the boundary } \partial \Omega \\ v_{C R}(z) & \text { if } z \text { is the midpoint of an edge } E \in \mathcal{E}(\Omega), \\ v_{\min }(z) & \text { if } z \in \mathcal{N}(\Omega)\end{cases}
$$

where the average $v_{\min }(z)$ in the interior node $z \in \mathcal{N}(\Omega)$ in the coarse triangulation $\mathcal{T}$ is determined locally on nodal patches $\omega_{z}^{*}$ covered by the triangles $\mathcal{T}^{*}(z):=\{T \in$ $\left.\mathcal{T}^{*} \mid z \in \mathcal{N}(T)\right\}$ of the red-refined triangulation $\mathcal{T}^{*}$ of Figure 3.1. Let

$$
W_{z}:=\left\{w \in P_{1}\left(\mathcal{T}^{*}(z)\right) \cap C\left(\bar{\omega}_{z}^{*}\right) \mid w=v_{C R} \text { on } \partial \omega_{z}^{*}\right\}
$$

denote the one-dimensional piecewise affine space of continuous functions on $\omega_{z}^{*}$ with prescribed boundary values on $\partial \omega_{z}^{*}$. The function $v_{\min }$ in $W_{z}$ is the unique minimizer of

$$
\min _{w \in W_{z}} \sum_{T \in \mathcal{T}^{*}(z)}\left\|\nabla\left(v_{C R}-w\right)\right\|_{L^{2}(T)}^{2}
$$

Lemma 3.4. Any Crouzeix-Raviart function $v_{C R} \in C R_{0}^{1}(\mathcal{T})$ with its jump of the tangential derivative $\left[\partial v_{C R} / \partial s\right]_{E}$ across an edge $E$ satisfies

$$
\left\|v_{C R}-\mathcal{I}_{C M} v_{C R}\right\|_{N C}^{2} \lesssim \sum_{E \in \mathcal{E}} h_{E}\left\|\left[\partial v_{C R} / \partial s\right]_{E}\right\|_{L^{2}(E)}^{2} \lesssim \min _{v \in V}\left\|v_{C R}-v\right\|_{N C}^{2} .
$$

Proof. The design of the interpolant $\mathcal{I}_{C M}$ shows that $\left\|\nabla\left(v_{C R}-\mathcal{I}_{C M} v_{C R}\right)\right\|_{L^{2}\left(T_{4}\right)}^{2}=0$ for those centred triangles $T_{4} \in \mathcal{T}^{*}$ with all three nodes of $T_{4}$ as midpoints of edges in the coarse triangulation $\mathcal{T}$. Let $z \in \mathcal{N}$ denote some node of $\mathcal{T}$ and set $\mathcal{E}^{*}(z):=\left\{F \in \mathcal{E}^{*} \mid z \in \mathcal{N}^{*}(F)\right\}$ for the smaller edges in the patch $\omega_{z}^{*}$ which share 
$z$ in the red-refined triangulation $\mathcal{T}^{*}$ of Figure 3.1. Consider the two semi-norms $\rho_{1}$ and $\rho_{2}$ defined, for all $\left.v_{C R} \in C R_{0}^{1}(\mathcal{T})\right|_{\omega_{z}^{*}}:=\left\{\left.v_{C R}\right|_{\omega_{z}^{*}}: v_{C R} \in C R_{0}^{1}(\mathcal{T})\right\}$, by

$$
\begin{aligned}
\rho_{1}\left(v_{C R}\right) & :=\| \nabla\left(v_{C R}-\mathcal{I}_{\left.C M v_{C R}\right) \|_{L^{2}\left(\omega_{z}^{*}\right)},}\right. \\
\rho_{2}\left(v_{C R}\right)^{2} & :=\sum_{F \in \mathcal{E}^{*}(z)} h_{F}\left\|\left[\partial v_{C R} / \partial s\right]_{F}\right\|_{L^{2}(F)}^{2} .
\end{aligned}
$$

In the first step one shows for some constant $C(z)$ that

$$
\rho_{1}\left(v_{C R}\right) \leq C(z) \rho_{2}\left(v_{C R}\right) \quad \text { for all }\left.v_{C R} \in C R_{0}^{1}(\mathcal{T})\right|_{\omega_{z}^{*}} .
$$

To do so, suppose that $\rho_{2}\left(v_{C R}\right)=0$. Then it holds that $\left.v_{C R}\right|_{\omega_{z}^{*}} \in C\left(\omega_{z}^{*}\right) \cap P_{1}\left(\omega_{z}^{*}\right)$. For an interior node $z$, it follows that $\left(\mathcal{I}_{C M} v_{C R}\right)(z)=v_{C R}(z)$ and so $\rho_{1}\left(v_{C R}\right)=$ 0 . For a boundary node $z, \rho_{2}\left(v_{C R}\right)=0$ implies $h_{F}\left\|\partial v_{C R} / \partial s\right\|_{L^{2}(F)}^{2}=0$ and so $v_{C R}$ vanishes along $F \in \mathcal{E}^{*}(z)$ with $F \subset \partial \Omega$. This implies $\mathcal{I}_{C M} v_{C R}(z)=0$ and so $\rho_{1}\left(v_{C R}\right)=0$. Hence, in either case $\rho_{2}\left(v_{C R}\right)=0$ implies $\rho_{1}\left(v_{C R}\right)=0$. The equivalence-of-norms argument on the finite-dimensional vector space $\left.C R_{0}^{1}(\mathcal{T})\right|_{\omega_{z}^{*}}$ proves (3.5) with some constant $C(z)$.

The second step verifies that $C(z) \lesssim 1$ with some standard scaling argument; hence the details are omitted.

In step three, the sum of all estimates (3.5) and the fact that $v_{C R}$ equals $\mathcal{I}_{C M} v_{C R}$ on all centred triangles in the red-refinement $\mathcal{T}^{*}$, show that

$$
\begin{aligned}
\left\|v_{C R}-\mathcal{I}_{C M} v_{C R}\right\|_{N C}^{2} & =\sum_{z \in \mathcal{N}}\left\|\nabla\left(v_{C R}-\mathcal{I}_{C M} v_{C R}\right)\right\|_{L^{2}\left(\omega_{z}^{*}\right)}^{2} \\
& \leq\left(\max _{z \in \mathcal{N}} C(z)\right) \sum_{E \in \mathcal{E}} h_{E}\left\|\left[\partial v_{C R} / \partial s\right]_{E}\right\|_{L^{2}(E)}^{2} .
\end{aligned}
$$

This concludes the proof of the first inequality.

The second inequality

$$
\sum_{E \in \mathcal{E}} h_{E}\left\|\left[\partial v_{C R} / \partial s\right]_{E}\right\|_{L^{2}(E)}^{2} \lesssim \min _{v \in V}\left\|v_{C R}-v\right\|_{N C}^{2}
$$

can be found in the context of efficiency of a posteriori error estimates for nonconforming schemes DDPV96, CELH12.

Lemma 3.5. $\mathcal{I}_{C M}: C R_{0}^{1}(\mathcal{T}) \rightarrow P_{1}\left(\mathcal{T}^{*}\right) \cap C_{0}(\Omega)$ is linear and uniformly bounded in the sense that

$$
\left\|\mathcal{I}_{C M}\right\|:=\sup _{v_{C R} \in C R_{0}^{1}(\mathcal{T}) \backslash\{0\}}\left\|\mathcal{I}_{C M} v_{C R}\right\| /\left\|v_{C R}\right\|_{N C} \lesssim 1 .
$$

Proof. The critical value $v_{\min }(z)$ of the minimising function $v_{\min } \in P_{1}\left(\mathcal{T}^{*}(z)\right)$ of (3.4) for an interior node $z \in \mathcal{N}(\Omega)$ is computed from the one-dimensional linear equation obtained from the optimality condition: The piecewise affine nodal basis function $\varphi_{z}^{*}$ associated with the node $z \in \mathcal{N}^{*}$ in the refined triangulation $\mathcal{T}^{*}$ satisfies

$$
\sum_{T \in \mathcal{T}^{*}(z)} \int_{T} \nabla \varphi_{z}^{*} \cdot \nabla\left(v_{\min }-v_{C R}\right) d x=0 .
$$

(This follows from the implementation of the boundary values on $\partial \omega_{z}^{*}$ and the ansatz of the remaining $v_{\min }(z) \varphi_{z}^{*}$.) This design shows that $\mathcal{I}_{C M}: C R_{0}^{1}(\mathcal{T}) \rightarrow$ 
$P_{1}\left(\mathcal{T}^{*}\right) \cap C_{0}(\Omega)$ is a linear operator. Lemma 3.4 plus some triangle inequality shows the boundedness of $\mathcal{I}_{C M}$ : Indeed, any $v_{C R} \in C R_{0}^{1}(\mathcal{T})$ satisfies

$$
\begin{aligned}
\left\|\mathcal{I}_{C M} v_{C R}\right\| & \leq\left\|v_{C R}-\mathcal{I}_{C M} v_{C R}\right\|_{N C}+\left\|v_{C R}\right\|_{N C} \\
& \lesssim \min _{v \in V}\left\|v_{C R}-v\right\|_{N C}+\left\|v_{C R}\right\|_{N C} \lesssim\left\|v_{C R}\right\|_{N C} .
\end{aligned}
$$

Lemma 3.6 (Upper bound). The conforming interpolation $\mathcal{I}_{C M} v_{C R} \in V_{C}\left(\mathcal{T}^{*}\right)$ of any nonconforming function $v_{C R} \in C R_{0}^{1}(\mathcal{T})$, which is normalised by $\left\|v_{C R}\right\|=1$, satisfies

$$
\lambda_{1} \leq R\left(\mathcal{I}_{C M} v_{C R}\right) .
$$

Proof. Since $\left\|v_{C R}\right\|=1,\left(\mathcal{I}_{C M} v_{C R}\right)(\operatorname{mid}(E))=v_{C R}(\operatorname{mid}(E)) \neq 0$ for at least one edge $E \in \mathcal{E}$. Hence, $\mathcal{I}_{C M} v_{C R} \not \equiv 0$. Therefore, the assertion follows immediately from the continuous Rayleigh-Ritz principle without any extra condition.

Example 3.7. For the three triangulations of the unit square $\Omega=(0,1)^{2}$ depicted in Figure 1.1, the first exact eigenvalue reads $\lambda_{1}=2 \pi^{2}=19.7392$ and is smaller than the first discrete conforming eigenvalue $\lambda_{C, 1}=24$ from the related one-dimensional algebraic eigenvalue problem for the criss-cross and the union-jack triangulations. The criss and the criss-cross triangulations of Figure 1.1 lead to the discrete nonconforming eigenvalue $\lambda_{C R, 1}=24$. The nonconforming eigenvalue approximation of the smallest eigenvalue for the union-jack triangulation reads $\lambda_{C R, 1}=18.3344$ up to some truncation error of finite machine precision from the iterative algebraic eigenvalue solver and is empirically below the exact eigenvalue. Theorem 3.1 leads to the guaranteed error bounds (1.3). Note that for the union-jack pattern, the proposed conforming interpolation on the red-refined triangulation $\mathcal{T}^{*}$ provides an upper bound which is strictly smaller than the conforming eigenvalue $\lambda_{C, 1}=24$ for the coarse mesh $\mathcal{T}$.

Since the algebraic eigenvalue problems are solved iteratively, the algebraic eigenvalue error has to be considered as well. The algebraic eigenvalue problem reads

$$
\mathbf{A} \mathbf{u}_{C R}=\lambda_{C R} \mathbf{B} \mathbf{u}_{C R}
$$

for the coefficient vector $\mathbf{u}_{C R} \equiv\left(u_{C R}(\operatorname{mid}(E)): E \in \mathcal{E}(\Omega)\right)$ of the discrete solution

$$
u_{C R}=\sum_{E \in \mathcal{E}(\Omega)} \mathbf{u}_{C R}(E) \psi_{E}
$$

for the edge-oriented basis $\left(\psi_{E} \mid E \in \mathcal{E}(\Omega)\right)$ of $C R_{0}^{1}(\mathcal{T})$. Set $\|\mathbf{x}\|_{M}:=\sqrt{\mathbf{x}^{T} M \mathbf{x}}$ for some SPD matrix $M$.

Lemma 3.8 ([ar98, Theorem 15.9.1]). Let $\left(\tilde{\lambda}_{C R}, \tilde{\mathbf{u}}_{C R}\right)$ be an approximated algebraic eigenpair such that $\tilde{\lambda}_{C R}$ is closer to some $\lambda_{C R}$ than to any other discrete eigenvalue. Suppose that the coefficient vector $\tilde{\mathbf{u}}_{C R}$ is normalised with respect to $\mathbf{B}$, $\left\|\mathbf{B} \tilde{\mathbf{u}}_{C R}\right\|_{\mathbf{B}^{-1}}=\left\|\tilde{\mathbf{u}}_{C R}\right\|_{\mathbf{B}}=1$. Then the algebraic residual $\mathbf{r}:=\mathbf{A} \tilde{\mathbf{u}}_{C R}-\tilde{\lambda}_{C R} \mathbf{B} \tilde{\mathbf{u}}_{C R}$ satisfies

$$
\left|\lambda_{C R}-\tilde{\lambda}_{C R}\right| \leq\|\mathbf{r}\|_{\mathbf{B}^{-1}} .
$$

Remark 3.9. The local mass matrix of the CR-NCFEM for some $T \in \mathcal{T}$ equals $|T| / 3$ times the $3 \times 3$ identity matrix $I_{3 \times 3}$. Hence, the global mass matrix $\mathbf{B}$ is diagonal and the residual norm $\|\mathbf{r}\|_{\mathbf{B}^{-1}}$ of the error bound is directly computable. 
Proof of Theorem 3.1. Lemma 3.8 and the monotonicity of $t /\left(1+\kappa^{2} H^{2} t\right)$ in $t>$ 0 allows us to formally replace $\lambda_{C R, 1}$ in Theorem 3.2 with $\tilde{\lambda}_{C R, 1}-\|\mathbf{r}\|_{\mathbf{B}^{-1}}$ for $\tilde{\lambda}_{C R, 1}>\|\mathbf{r}\|_{\mathbf{B}^{-1}}$ which proves the lower bound. The upper bound is proven in Lemma 3.6

Example 3.10. Since the iterative solution of the underlying discrete algebraic eigenvalue problem dominates the overall computational costs in general, the truncation error in the iterative solution may be much larger than machine precision. For example, the Rayleigh-quotient for the starting vector $(1, \ldots, 1) \in \mathbb{R}^{8}$ of the union-jack triangulation of Figure 1.1] (discussed also in Example 3.7) yields the nonconforming eigenvalue approximation $\tilde{\lambda}_{C R, 1}=24$ and the corresponding guaranteed bounds

$$
6.9360 \leq \lambda_{1} \leq 24
$$

This is competitive with the bounds (1.3) from much more expensive eigenvalue computations.

\section{EFFICIENCY FOR GRADED MESHES}

This section is devoted to the efficiency of the eigenvalue estimate of Theorem 3.1 with the difference of its upper and lower bounds

$$
\eta:=R\left(\mathcal{I}_{C M} \tilde{u}_{C R, 1}\right)-\frac{\tilde{\lambda}_{C R, 1}-\|\mathbf{r}\|_{\mathbf{B}^{-1}}}{1+\kappa^{2}\left(\tilde{\lambda}_{C R, 1}-\|\mathbf{r}\|_{\mathbf{B}^{-1}}\right) H^{2}} .
$$

Efficiency means that this length $\eta$ of the interval is bounded in terms of the error and will be proven in the following theorem for the class of graded meshes. (Graded meshes will be defined in the second half of this section.)

Theorem 4.1. For all graded meshes the estimate of Theorem 3.1 is efficient in the sense that the difference $\eta$ of the upper and lower bounds satisfies

$$
\begin{aligned}
\eta \lesssim & \left(1+H^{2} \tilde{\lambda}_{C R, 1}\right)\left\|u_{1}-\tilde{u}_{C R, 1}\right\|_{N C}^{2}+H^{2}\left(\left(\lambda_{1}-\lambda_{C R, 1}\right)^{2}+\lambda_{1} \lambda_{C R, 1}\left\|u_{1}-u_{C R, 1}\right\|^{2}\right) \\
& +\left|\lambda_{1}-\tilde{\lambda}_{C R, 1}\right|+\left\|\mathbf{A}\left(\mathbf{u}_{C R, 1}-\tilde{\mathbf{u}}_{C R, 1}\right)\right\|_{\mathbf{B}^{-1}} \\
& +\lambda_{C R, 1}\left\|u_{C R, 1}-\tilde{u}_{C R, 1}\right\|+\left|\lambda_{C R, 1}-\tilde{\lambda}_{C R, 1}\right| .
\end{aligned}
$$

The remaining parts of this section are devoted to the proof of Theorem 4.1. The first results hold on arbitrary shape-regular meshes.

Lemma 4.2. The difference $\eta$ from (4.1) of the lower and upper eigenvalue bounds satisfies

$$
\begin{aligned}
\eta \lesssim & \left(1+H^{2} \tilde{\lambda}_{C R, 1}\right)\left\|u_{1}-\tilde{u}_{C R, 1}\right\|_{N C}^{2}+\left|\lambda_{1}-\tilde{\lambda}_{C R, 1}\right|+\tilde{\lambda}_{C R, 1}^{2} H^{2} \\
& +\left\|\mathbf{A}\left(\mathbf{u}_{C R, 1}-\tilde{\mathbf{u}}_{C R, 1}\right)\right\|_{\mathbf{B}^{-1}}+\lambda_{C R, 1}\left\|u_{C R, 1}-\tilde{u}_{C R, 1}\right\|+\left|\lambda_{C R, 1}-\tilde{\lambda}_{C R, 1}\right| .
\end{aligned}
$$

Proof. Some preliminary manipulations in step one of this proof show that

$$
\begin{aligned}
& \eta=R\left(\mathcal{I}_{C M} \tilde{u}_{C R, 1}\right)-\frac{\tilde{\lambda}_{C R, 1}}{1+\kappa^{2}\left(\tilde{\lambda}_{C R, 1}-\|\mathbf{r}\|_{\mathbf{B}^{-1}}\right) H^{2}}+\frac{\|\mathbf{r}\|_{\mathbf{B}^{-1}}}{1+\kappa^{2}\left(\tilde{\lambda}_{C R, 1}-\|\mathbf{r}\|_{\mathbf{B}^{-1}}\right) H^{2}} \\
& \leq R\left(\mathcal{I}_{C M} \tilde{u}_{C R, 1}\right)-\lambda_{1}+\left|\lambda_{1}-\tilde{\lambda}_{C R, 1}\right|+\tilde{\lambda}_{C R, 1} \frac{\kappa^{2}\left(\tilde{\lambda}_{C R, 1}-\|\mathbf{r}\|_{\mathbf{B}^{-1}}\right) H^{2}}{1+\kappa^{2}\left(\tilde{\lambda}_{C R, 1}-\|\mathbf{r}\|_{\mathbf{B}^{-1}}\right) H^{2}}+\|\mathbf{r}\|_{\mathbf{B}^{-1}} \\
& \leq R\left(\mathcal{I}_{C M} \tilde{u}_{C R, 1}\right)-\lambda_{1}+\left|\lambda_{1}-\tilde{\lambda}_{C R, 1}\right|+\tilde{\lambda}_{C R, 1}^{2} \kappa^{2} H^{2}+\|\mathbf{r}\|_{\mathbf{B}^{-1}}
\end{aligned}
$$


Step two will be the proof of

$$
R\left(\mathcal{I}_{C M} \tilde{u}_{C R, 1}\right)-\lambda_{1} \lesssim\left(1+H^{2} \tilde{\lambda}_{C R, 1}\right)\left\|u_{1}-\tilde{u}_{C R, 1}\right\|_{N C}^{2} .
$$

Elementary algebra reveals for $\tilde{v}_{C}:=\mathcal{I}_{C M} \tilde{u}_{C R, 1} /\left\|\mathcal{I}_{C M} \tilde{u}_{C R, 1}\right\|$ that

$$
R\left(\mathcal{I}_{C M} \tilde{u}_{C R, 1}\right)-\lambda_{1}=\left\|\tilde{v}_{C}\right\|^{2}-\left\|u_{1}\right\|^{2}=\left\|u_{1}-\tilde{v}_{C}\right\|^{2}+2 a\left(u_{1}, \tilde{v}_{C}-u_{1}\right) .
$$

Since $V_{C}\left(\mathcal{T}^{*}\right) \subset V$ and $\left\|u_{1}\right\|=1=\left\|\tilde{v}_{C}\right\|$ it follows that

$$
2 a\left(u_{1}, \tilde{v}_{C}-u_{1}\right)=-2 \lambda_{1}+2 \lambda_{1} b\left(u_{1}, \tilde{v}_{C}\right)=-\lambda_{1}\left\|u_{1}-\tilde{v}_{C}\right\|^{2} \leq 0 .
$$

This shows that

$$
R\left(\mathcal{I}_{C M} \tilde{u}_{C R, 1}\right)-\lambda_{1} \leq 2\left\|\tilde{u}_{C R, 1}-\tilde{v}_{C}\right\|_{N C}^{2}+2\left\|u_{1}-\tilde{u}_{C R, 1}\right\|_{N C}^{2} .
$$

The Young inequality leads to

$$
\begin{aligned}
\left\|\tilde{u}_{C R, 1}-\tilde{v}_{C}\right\|_{N C}^{2} & =\left\|\tilde{u}_{C R, 1}-\mathcal{I}_{C M} \tilde{u}_{C R, 1}+\mathcal{I}_{C M} \tilde{u}_{C R, 1}\left(1-1 /\left\|\mathcal{I}_{C M} \tilde{u}_{C R, 1}\right\|\right)\right\|_{N C}^{2} \\
& \leq 2\left\|\tilde{u}_{C R, 1}-\mathcal{I}_{C M} \tilde{u}_{C R, 1}\right\|_{N C}^{2}+2\left(\left\|\mathcal{I}_{C M} \tilde{u}_{C R, 1}\right\|-1\right)^{2} R\left(\mathcal{I}_{C M} \tilde{u}_{C R, 1}\right) .
\end{aligned}
$$

Since $\left\|\tilde{u}_{C R, 1}\right\|=1$, an inverse triangle inequality shows

$$
\left(\left\|\mathcal{I}_{C M} \tilde{u}_{C R, 1}\right\|-1\right)^{2}=\left(\left\|\mathcal{I}_{C M} \tilde{u}_{C R, 1}\right\|-\left\|\tilde{u}_{C R, 1}\right\|\right)^{2} \leq\left\|\tilde{u}_{C R, 1}-\mathcal{I}_{C M} \tilde{u}_{C R, 1}\right\|^{2} .
$$

Note that $\left.\left(\tilde{u}_{C R, 1}-\mathcal{I}_{C M} \tilde{u}_{C R, 1}\right)\right|_{T_{4}} \equiv 0$ on each centred triangle $T_{4}$ in $\mathcal{T}^{*}$. For the remaining triangles $T \in \mathcal{T}^{*}$ of the patches $\omega_{z}^{*}$ for nodes $z \in \mathcal{N}(\Omega)$, it holds that $\left.\left(\tilde{u}_{C R, 1}-\mathcal{I}_{C M} \tilde{u}_{C R, 1}\right)\right|_{E} \equiv 0$ on the edges $E$ with $E \subset \partial \omega_{z}^{*}$. Hence, the Friedrich's inequality shows, for those triangles, that

$$
\left\|\tilde{u}_{C R, 1}-\mathcal{I}_{C M} \tilde{u}_{C R, 1}\right\|_{L^{2}(T)} \leq h_{T}\left\|\nabla\left(\tilde{u}_{C R, 1}-\mathcal{I}_{C M} \tilde{u}_{C R, 1}\right)\right\|_{L^{2}(T)} .
$$

The summation over all triangles yields

$$
\left\|\tilde{u}_{C R, 1}-\mathcal{I}_{C M} \tilde{u}_{C R, 1}\right\| \lesssim H\left\|\tilde{u}_{C R, 1}-\mathcal{I}_{C M} \tilde{u}_{C R, 1}\right\|_{N C} .
$$

The remaining term $H^{2} R\left(\mathcal{I}_{C M} \tilde{u}_{C R, 1}\right)$ is bounded by $16 H^{2} \tilde{\lambda}_{C R, 1}$ because of the uniform boundedness of $\mathcal{I}_{C M}$ in Lemma 3.5 and the inequality of the discrete norms $\left\|\tilde{u}_{C R, 1}\right\|^{2} \leq 16\left\|\mathcal{I}_{C M} \tilde{u}_{C R, 1}\right\|^{2}$. The proof of the latter estimate considers the centred triangle $T_{4}$ of the fine triangulation $\mathcal{T}^{*}$ with $\left.\left(\mathcal{I}_{C M} \tilde{u}_{C R, 1}\right)\right|_{T_{4}}=\left.\tilde{u}_{C R, 1}\right|_{T_{4}}$. Set $x:=$ $\left(\tilde{u}_{C R, 1}\left(\operatorname{mid}\left(E_{j}\right)\right)\right)_{j=1,2,3} \in \mathbb{R}^{3}$ of the three edges $E_{1}, E_{2}, E_{3}$ of $T$ and compute (with the Rayleigh quotient $\geq 1$ of the displayed $3 \times 3$ matrix)

$$
\left\|\mathcal{I}_{C M} \tilde{u}_{C R, 1}\right\|_{L^{2}\left(T_{4}\right)}^{2}=\frac{|T|}{48} x^{T}\left(\begin{array}{ccc}
2 & 1 & 1 \\
1 & 2 & 1 \\
1 & 1 & 2
\end{array}\right) x \geq \frac{|T|}{48} x \cdot x=\left\|\tilde{u}_{C R, 1}\right\|_{L^{2}(T)}^{2} / 16 .
$$

Finally, the estimate

$$
\left\|\tilde{u}_{C R, 1}-\mathcal{I}_{C M} \tilde{u}_{C R, 1}\right\|_{N C}^{2} \lesssim \sum_{E \in \mathcal{E}} h_{E}\left\|\left[\partial \tilde{u}_{C R, 1} / \partial s\right]_{E}\right\|_{L^{2}(E)}^{2} \lesssim \min _{v \in V}\left\|\tilde{u}_{C R, 1}-v\right\|_{N C}^{2}
$$

from Lemma 3.4 concludes the proof of (4.3) in step two.

Step three will be the proof of

$$
\begin{aligned}
\|\mathbf{r}\|_{\mathbf{B}^{-1}} \leq & \left\|\mathbf{A}\left(\mathbf{u}_{C R, 1}-\tilde{\mathbf{u}}_{C R, 1}\right)\right\|_{\mathbf{B}^{-1}} \\
& +\lambda_{C R, 1}\left\|u_{C R, 1}-\tilde{u}_{C R, 1}\right\|+\left|\lambda_{C R, 1}-\tilde{\lambda}_{C R, 1}\right| .
\end{aligned}
$$




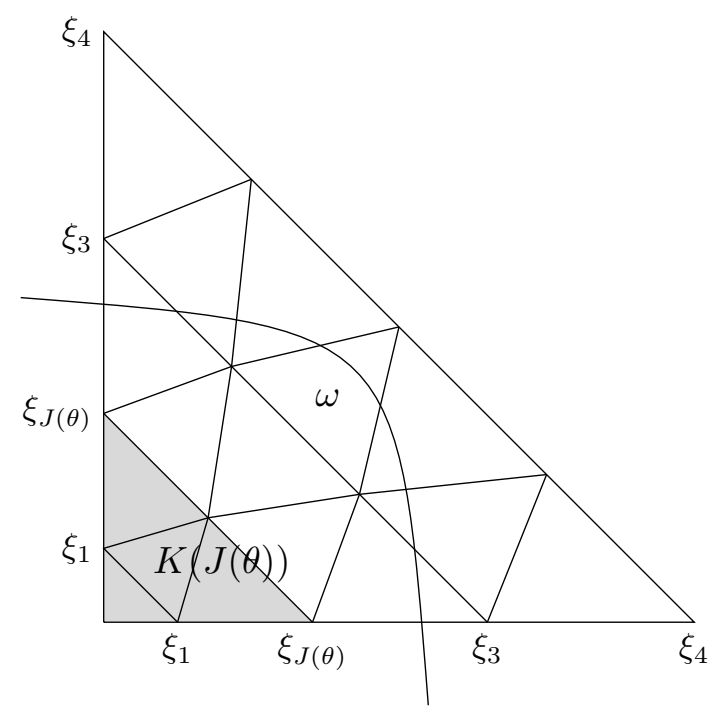

FIGURE 4.1. Reference triangle $T_{\text {ref }}$ with $3 / 2$-graded sub-triangles for $N=4$.

The definition of the algebraic residual $\mathbf{r}:=\mathbf{A} \tilde{\mathbf{u}}_{C R, 1}-\tilde{\lambda}_{C R, 1} \mathbf{B} \tilde{\mathbf{u}}_{C R, 1}$ plus the triangle inequality yield

$$
\begin{aligned}
\|\mathbf{r}\|_{\mathbf{B}^{-1}}= & \| \mathbf{A} \tilde{\mathbf{u}}_{C R, 1}-\mathbf{A} \mathbf{u}_{C R, 1}+\lambda_{C R, 1} \mathbf{B} \mathbf{u}_{C R, 1}-\lambda_{C R, 1} \mathbf{B} \tilde{\mathbf{u}}_{C R, 1} \\
& +\lambda_{C R, 1} \mathbf{B} \tilde{\mathbf{u}}_{C R, 1}-\tilde{\lambda}_{C R, 1} \mathbf{B} \tilde{\mathbf{u}}_{C R, 1} \|_{\mathbf{B}^{-1}} .
\end{aligned}
$$

This and the triangle inequality prove (4.5) in step three.

Step four is the finish of the proof. Indeed, the combination of (4.2)- (4.5) concludes the proof of Lemma 4.2 .

The following estimate is proven with the same arguments as in the conforming case and is reported in DDP12 for the nonconforming CR-NCFEM.

Lemma 4.3 ([DDP12] $)$. Let $\left(\lambda_{C R}, u_{C R}\right) \in \mathbb{R} \times C R_{0}^{1}(\mathcal{T})$ be a discrete eigenpair of the eigenpair $(\lambda, u)$, then it holds that

$$
\left\|h_{\mathcal{T}} \lambda_{C R} u_{C R}\right\|^{2} \lesssim\left\|u-u_{C R}\right\|_{N C}^{2}+H^{2}\left(\left(\lambda-\lambda_{C R}\right)^{2}+\lambda \lambda_{C R}\left\|u-u_{C R}\right\|^{2}\right) .
$$

The second half of this section concerns the somewhat surprising result of Theorem 4.4 for graded meshes which are described in the following.

Given a polygonal domain with a coarse triangulation $\mathcal{T}_{0}$ into triangles called macro elements (which specify the geometry), the domain $\Omega$ will be covered by piecewise affine images of the graded mesh on the reference triangle $T_{\text {ref }}$ with vertices $(0,0),(1,0)$, and $(0,1)$. Provided the coarse triangulation satisfies the condition that each triangle has at most one vertex as a corner of $\partial \Omega$, then the grading parameter $\beta$ can be different for each such corner of $\partial \Omega$ and $\beta:=1$ for all those macro triangles without a vertex at a corner of $\partial \Omega$. One verifies directly that the structured mesh is a (shape) regular triangulation. On each element $K \in \mathcal{T}_{0}$, the mesh of the reference triangle is obtained by an affine transformation. The graded mesh on the macro element $T_{\text {ref }}$ of Figure 4.1 is generated as follows: Given some grading parameter $\beta>0$ and given an integer $N \geq 2$, set $\xi_{j}:=(j / N)^{\beta}$ and draw line 
segments aligned to the anti-diagonal through $\left(0, \xi_{j}\right)$ and $\left(\xi_{j}, 0\right)$ for $j=0,1, \ldots, N$. Each of these line segments is divided into $j$ uniform edges and so define the set of nodes $(0,0)$ and $(j-k, k) \xi_{j} / j$ for $k=0, \ldots, j$ and $j=1, \ldots, N$. The triangles are then given by the vertices $\xi_{j} / j(j-k, k)$ and $\xi_{j} / j(j-k-1, k+1)$ aligned with anti-diagonal and the vertex $\xi_{j-1} /(j-1)(j-k-1, k)$ on the finer segment and $\xi_{j+1} /(j+1)(j-k, k+1)$ on the coarser neighbouring segment. The smallest triangle reads $\operatorname{conv}\left\{(0,0),\left(0, \xi_{1}\right),\left(\xi_{1}, 0\right)\right\}$ with diameter $\sqrt{2} \xi_{1} \approx N^{-\beta}$. The largest triangles have diameter $H \approx N^{-1}$.

Theorem 4.4. Any function $f \in L^{2}(\Omega) \backslash\{0\}$ and any graded triangulation $\mathcal{T}$ of $\Omega$ satisfy

$$
\left\|h_{\mathcal{T}} f\right\| \approx 1 / N .
$$

The equivalence constant $C(f)$ in the assertion $1 / N \leq C(f)\left\|h_{\mathcal{T}} f\right\|$ depends on $f$.

Proof. The first inequality follows from

$$
\left\|h_{\mathcal{T}} f\right\| \leq H\|f\| \approx\|f\| / N .
$$

To verify the reverse inequality, consider one triangle $K \in \mathcal{T}_{0}$. Some affine diffeomorphism (which depends only on $\mathcal{T}_{0}$ ) maps $K$ onto $T_{\text {ref }}$ and some transformation shows that it suffices to verify the assertion on $T_{\text {ref }}$. Without loss of generality, let $f \in L^{2}\left(T_{\text {ref }}\right) \backslash\{0\}$ satisfy $|f| \geq \varepsilon>0$ on a set $\omega$ of measure $|\omega|>0$. Let the volume fraction $\theta:=|\omega| /\left|T_{\text {ref }}\right|$ of $\omega$ in $T_{\text {ref }}$ be fixed and consider the question where $\omega \subset T_{\text {ref }}$ of fixed area $|\omega|=\theta / 2$ may be located to minimise the term $\int_{T_{\text {ref }} \cap \omega} h_{\mathcal{T}}^{2} d x$. Figure 4.1 illustrates the situation where $\omega$ is placed where $h_{T}$ is small. In the end, one deduces that for some index $J(\theta)$ (which is maximal with $\xi_{J(\theta)}^{2} \leq \theta$ ), the minimising set $\omega$ includes the sub-triangle $K(J(\theta)):=\operatorname{conv}\left\{(0,0),\left(\xi_{J(\theta)}, 0\right),\left(0, \xi_{J(\theta)}\right)\right\}$ and the induced sub-triangulation $\mathcal{T}(K(J(\theta)))$. Hence,

$$
\left\|h_{\mathcal{T}}\right\|_{L^{2}(K(J(\theta))} \leq\left\|h_{\mathcal{T}}\right\|_{L^{2}(\omega)} \leq \frac{1}{\varepsilon}\left\|h_{\mathcal{T} f}\right\|_{L^{2}(\omega)} \leq \frac{1}{\varepsilon}\left\|h_{\mathcal{T}} f\right\|_{L^{2}(\Omega)} .
$$

Thus it remains to prove that

$$
N^{-1} \lesssim\left\|h_{\mathcal{T}}\right\|_{L^{2}(K(J(\theta))}
$$

Since the $j$-th diagonal layer consists of $2 j-1$ triangles, it holds that

$$
\sum_{j=1}^{J(\theta)}(2 j-1)\left(\frac{j^{\beta}-(j-1)^{\beta}}{N^{\beta}}\right)^{4} \lesssim \sum_{T \in \mathcal{T}(K(J(\theta)))} \int_{T} h_{T}^{2} d x
$$

The binomial expansion shows

$$
\sum_{j=1}^{J(\theta)}\left(\frac{j^{\beta}-(j-1)^{\beta}}{N^{\beta}}\right)^{4} \approx \sum_{j=1}^{J(\theta)}\left(\frac{j^{\beta-1} \beta}{N^{\beta}}\right)^{4}
$$

This leads to

$$
\frac{\beta^{4}}{N^{2}} \sum_{j=1}^{J(\theta)}\left(\frac{j}{N}\right)^{4 \beta-3} N^{-1}=\sum_{j=1}^{J(\theta)} j\left(\frac{j^{\beta-1} \beta}{N^{\beta}}\right)^{4} \lesssim \sum_{j=1}^{J(\theta)}(2 j-1)\left(\frac{j^{\beta}-(j-1)^{\beta}}{N^{\beta}}\right)^{4} .
$$


Since $J(\theta)=N \sqrt[2 \beta]{2|K(J(\theta))|}$, the sum on the right-hand side is a Riemann sum over the interval $[0, \sqrt[2 \beta]{2|K(J(\theta))|}$. Since $\beta \geq 1$,

$$
\begin{aligned}
\frac{\beta^{4}}{N^{2}} \sum_{j=1}^{J(\theta)}\left(\frac{j}{N}\right)^{4 \beta-3} N^{-1} & \approx \frac{\beta^{4}}{N^{2}} \int_{0}^{\sqrt[2 \beta]{2|K(J(\theta))|}} x^{4 \beta-3} d x \\
& =\frac{\beta^{4}(2|K(J(\theta))|)^{(2 \beta-1) / \beta}}{N^{2}(4 \beta-2)} \approx \frac{1}{N^{2}}
\end{aligned}
$$

This proves the assertion for $N \geq N_{0}$ and sufficiently large $N_{0}$ so that $J(\theta) \geq 1$. For $1 \leq N \leq N_{0}, N\left\|h_{\mathcal{T}} f\right\| \geq N^{1-\beta}\|f\|$ is bounded from below in terms of $N_{0}$. This concludes the proof for all $N \in \mathbb{N}$.

Proof of Theorem 4.1. The assertion follows from Lemma 4.2, Theorem 4.4, and Lemma 4.3 .

\section{ERROR BOUNDS FOR HIGHER EIGENVALUES}

This section is devoted to some computable lower bounds of higher eigenvalues. It is emphasised that $\lambda_{J}$ could be a multiple eigenvalue and $\lambda_{J}$ could even be a part of a cluster without any separation (on the continuous level); cf. Example 5.3 below. However, any clustering of discrete eigenvalues may have some disastrous effect on the smallness of the discrete residual $\mathbf{r}$ in the algebraic eigenvalue problem.

Theorem 5.1. Suppose that the separation condition $H<(\sqrt{1+1 / J}-1) /\left(\kappa \lambda_{J}^{1 / 2}\right)$ holds for the J-th exact eigenvalue $\lambda_{J}$. Let $\left(\tilde{\lambda}_{C R, J}, \tilde{u}_{C R, J}\right) \in \mathbb{R} \times C R_{0}^{1}(\mathcal{T})$ with $\left\|\tilde{u}_{C R, J}\right\|_{L^{2}(\Omega)}=1$ and algebraic residual $\mathbf{r}:=\mathbf{A} \tilde{\mathbf{u}}_{C R, J}-\tilde{\lambda}_{C R, J} \mathbf{B} \tilde{\mathbf{u}}_{C R, J}$ approximate the J-th eigenpair $\left(\lambda_{J}, u_{J}\right)$. Suppose separation of $\tilde{\lambda}_{C R, J}$ from the remaining discrete spectrum in the sense that $\tilde{\lambda}_{C R, J}$ is closer to the discrete eigenvalue $\lambda_{C R, J}$ than to any other discrete eigenvalues and that $\|\mathbf{r}\|_{\mathbf{B}^{-1}}<\tilde{\lambda}_{C R, J}$. Then it holds that

$$
\frac{\tilde{\lambda}_{C R, J}-\|\mathbf{r}\|_{\mathbf{B}^{-1}}}{1+\kappa^{2}\left(\tilde{\lambda}_{C R, J}-\|\mathbf{r}\|_{\mathbf{B}^{-1}}\right) H^{2}} \leq \lambda_{J} \leq \max _{\xi \in \mathbb{R}^{J} \backslash\{0\}} R\left(\sum_{j=1}^{J} \xi_{j} \mathcal{I}_{C M} \tilde{u}_{C R, j}\right) .
$$

The difference of the upper and lower bounds

$$
\eta_{J}:=\max _{\xi \in \mathbb{R}^{J} \backslash\{0\}} R\left(\sum_{j=1}^{J} \xi_{j} \mathcal{I}_{C M} \tilde{u}_{C R, j}\right)-\frac{\tilde{\lambda}_{C R, J}-\|\mathbf{r}\|_{\mathbf{B}^{-1}}}{1+\kappa^{2}\left(\tilde{\lambda}_{C R, J}-\|\mathbf{r}\|_{\mathbf{B}^{-1}}\right) H^{2}}
$$

is efficient in the sense that

$$
\begin{aligned}
\eta_{J} \lesssim & \left(1+H^{2} \max _{\xi \in \mathbb{R}^{J} \backslash\{0\}} R\left(\sum_{j=1}^{J} \xi_{j} \mathcal{I}_{C M} \tilde{u}_{C R, j}\right)\right) \max _{\xi \in \mathbb{R}^{J} \backslash\{0\}}\left\|u_{J}-\sum_{j=1}^{J} \xi_{j} \tilde{u}_{C R, j}\right\|_{N C} \\
& +\left|\lambda_{J}-\tilde{\lambda}_{C R, J}\right|+H^{2}\left(\left(\lambda_{J}-\lambda_{C R, J}\right)^{2}+\lambda_{J} \lambda_{C R, J}\left\|u_{J}-u_{C R, J}\right\|^{2}\right) \\
& +\left\|\mathbf{A}\left(\mathbf{u}_{C R, J}-\tilde{\mathbf{u}}_{C R, J}\right)\right\|_{\mathbf{B}^{-1}}+\lambda_{C R, J}\left\|u_{C R, J}-\tilde{u}_{C R, J}\right\|+\left|\lambda_{C R, J}-\tilde{\lambda}_{C R, J}\right| .
\end{aligned}
$$

The proofs start with the linear independence of nonconforming interpolants.

Lemma 5.2. Let $\left(u_{1}, \ldots, u_{J}\right)$ be some b-orthonormal basis of exact eigenvectors in $V$ for the exact first $J$ eigenvalues $0<\lambda_{1}<\lambda_{2} \leq \ldots \leq \lambda_{J}$ on the continuous 
level. For any global mesh-size $H<(\sqrt{1+1 / J}-1) /\left(\kappa \lambda_{J}^{1 / 2}\right)$, the nonconforming interpolants $\mathcal{I}_{N C} u_{1}, \ldots, \mathcal{I}_{N C} u_{J}$ are linear independent.

Proof. For any $j=1, \ldots, J$, Theorem 2.1 shows

$$
\left\|u_{j}-\mathcal{I}_{N C} u_{j}\right\| \leq \kappa H\left\|u_{j}-\mathcal{I}_{N C} u_{j}\right\|_{N C} \leq \kappa H\left\|u_{j}\right\|_{N C} \leq \kappa H \lambda_{j}^{1 / 2}=: d_{j} .
$$

With the Kronecker $\delta_{j k}=1$ for $j=k$ and $\delta_{j k}=0$ for $j \neq k$, this implies

$$
\begin{aligned}
& \left|b\left(\mathcal{I}_{N C} u_{j}, \mathcal{I}_{N C} u_{k}\right)-\delta_{j k}\right|=\left|b\left(\mathcal{I}_{N C} u_{j}, \mathcal{I}_{N C} u_{k}\right)-b\left(u_{j}, u_{k}\right)\right| \\
& =\left|-b\left(u_{j}-\mathcal{I}_{N C} u_{j}, \mathcal{I}_{N C} u_{k}\right)-b\left(u_{j}, u_{k}-\mathcal{I}_{N C} u_{k}\right)\right| \\
& =\left|b\left(u_{j}-\mathcal{I}_{N C} u_{j}, u_{k}-\mathcal{I}_{N C} u_{k}\right)-b\left(u_{j}-\mathcal{I}_{N C} u_{j}, u_{k}\right)-b\left(u_{j}, u_{k}-\mathcal{I}_{N C} u_{k}\right)\right| \\
& \leq\left\|u_{j}-\mathcal{I}_{N C} u_{j} \mid\right\| u_{k}-\mathcal{I}_{N C} u_{k}\|+\| u_{j}-\mathcal{I}_{N C} u_{j}\|+\| u_{k}-\mathcal{I}_{N C} u_{k} \| \\
& \leq d_{j} d_{k}+d_{j}+d_{k} .
\end{aligned}
$$

Some calculations show that $H<(\sqrt{1+1 / J}-1) /\left(\kappa \lambda_{J}^{1 / 2}\right)$ leads to

$$
\max _{j=1}^{J}\left(\sum_{k=1}^{J}\left(d_{j} d_{k}+d_{j}+d_{k}\right)\right)<1 .
$$

The Gershgorin theorem shows that the eigenvalues of $\left(b\left(\mathcal{I}_{N C} u_{j}, \mathcal{I}_{N C} u_{k}\right)\right)_{j, k=1, \ldots, J}$ are all positive.

Proof of the lower bound in Theorem 5.1 for $\mathbf{r} \equiv 0$. Lemma 5.2 guarantees that

$$
\mathcal{I}_{N C} u_{1}, \ldots, \mathcal{I}_{N C} u_{J}
$$

are linearly independent. The Rayleigh-Ritz principle on the discrete level states that the discrete eigenvalue $\lambda_{C R, J}$ of number $J$ equals

$$
\lambda_{C R, J}=\min _{V_{J} \subset C R_{0}^{1}(\mathcal{T}), \operatorname{dim}\left(V_{J}\right)=J} \max _{v \in V_{J} \backslash\{0\}} R_{N C}(v) .
$$

Therein, the notation $\operatorname{dim}\left(V_{J}\right)=J$ abbreviates that the minimum is taken over all subspaces of $C R_{0}^{1}(\mathcal{T})$ of dimension $J$. Since $\mathcal{I}_{N C} u_{1}, \ldots, \mathcal{I}_{N C} u_{J}$ are linear independent, there exist some real coefficients $\xi_{1}, \ldots, \xi_{J}$ such that the Rayleigh quotient is maximised in $V_{J}:=\operatorname{span}\left\{\mathcal{I}_{N C} u_{1}, \ldots, \mathcal{I}_{N C} u_{J}\right\}$. This leads to

$$
\lambda_{C R, J} \leq R_{N C}\left(\sum_{j=1}^{J} \xi_{j} \mathcal{I}_{N C} u_{j}\right) .
$$

One may assume without loss of generality that

$$
\sum_{j=1}^{J} \xi_{j}^{2}=1
$$

Let $v:=\sum_{j=1}^{J} \xi_{j} u_{j}$ and observe that $\|v\|^{2}=\sum_{j=1}^{J} \xi_{j}^{2}=1$. Since $\nabla_{N C}\left(v-\mathcal{I}_{N C} v\right)$ is $L^{2}$ orthogonal to $\nabla_{N C} \mathcal{I}_{N C} v$, the Pythagoras theorem reads

$$
\left\|v-\mathcal{I}_{N C} v\right\|_{N C}^{2}+\left\|\mathcal{I}_{N C} v\right\|_{N C}^{2}=\|v\|^{2} .
$$

The orthogonality of the eigenfunctions shows

$$
\|v\|^{2}=\left\|\sum_{j=1}^{J} \xi_{j} u_{j}\right\|^{2}=\sum_{j=1}^{J} \xi_{j}^{2}\left\|u_{j}\right\|^{2}=\sum_{j=1}^{J} \xi_{j}^{2} \lambda_{j} .
$$


The combination of the aforementioned equalities results in

$$
\left\|v-\mathcal{I}_{N C} v\right\|_{N C}^{2}+\left\|\mathcal{I}_{N C} v\right\|_{N C}^{2}=\sum_{j=1}^{J} \xi_{j}^{2} \lambda_{j} \leq \lambda_{J} .
$$

Together with (5.3) in the form of

$$
\lambda_{C R, J}\left\|\mathcal{I}_{N C} v\right\|^{2} \leq\left\|\mathcal{I}_{N C} v\right\|_{N C}^{2},
$$

the previous estimate yields

$$
\left\|v-\mathcal{I}_{N C} v\right\|_{N C}^{2}+\lambda_{C R, J}\left\|\mathcal{I}_{N C} v\right\|^{2} \leq \lambda_{J} .
$$

Since $\|v\|^{2}=1$, the Cauchy inequality followed by the binomial expansion implies $1+\left\|v-\mathcal{I}_{N C} v\right\|^{2}-2\left\|v-\mathcal{I}_{N C} v\right\| \leq 1+\left\|v-\mathcal{I}_{N C} v\right\|^{2}-2 b\left(v-\mathcal{I}_{N C} v, v\right)=\left\|\mathcal{I}_{N C} v\right\|^{2}$.

Following the proof of Theorem 3.1 with the substitution of $u_{1}$ by $v$ eventually results in

$$
\frac{\lambda_{C R, J}}{1+\kappa^{2} \lambda_{C R, J} H^{2}} \leq \lambda_{J}
$$

Proof of the lower bound in Theorem 5.1 for $\mathbf{r} \not \equiv 0$. Lemma 3.8 and the monotonicity of $t /\left(1+\kappa^{2} H^{2} t\right)$ in $t>0$ allows the substitution of $\lambda_{C R}$ by $\tilde{\lambda}_{C R, J}-\|\mathbf{r}\|_{B^{-1}}$ for $\tilde{\lambda}_{C R, J}>\|\mathbf{r}\|_{B^{-1}}$.

Proof of the upper bound in Theorem [5.1. Let $\tilde{u}_{C R, 1}, \ldots, \tilde{u}_{C R, J}$ be the first $J$ approximated discrete orthonormal eigenfunctions. Since $\mathcal{I}_{C M} \tilde{u}_{C R, 1} \equiv \tilde{u}_{C R, 1}$ on each centred triangle of $\mathcal{T}^{*}$ with all vertices as midpoints of edges in $\mathcal{E}(\mathcal{T})$, the functions $\mathcal{I}_{C M} \tilde{u}_{C R, 1}, \ldots, \mathcal{I}_{C M} \tilde{u}_{C R, J}$ are linear independent. Thus, there exist some maximising coefficients $\xi_{j}$ with $\sum_{j=1}^{J} \xi_{j}^{2}=1$ such that

$$
\lambda_{J}=\min _{V_{J} \subset V, \operatorname{dim}\left(V_{J}\right)=J} \max _{v \in V_{J} \backslash\{0\}} R(v) \leq R\left(\sum_{j=1}^{J} \xi_{j} \mathcal{I}_{C M} \tilde{u}_{C R, j}\right) .
$$

Proof of efficiency in Theorem 5.1. The proof of efficiency of the difference of the upper and lower bounds in (5.1) follows from some modifications of the arguments of Lemmas 4.2, 4.3 and Theorem 4.4. Therefore the remaining parts of this proof only sketch the main steps. The arguments in (4.2) lead to

$$
\eta_{J} \leq \max _{\xi \in \mathbb{R}^{J} \backslash\{0\}} R\left(\sum_{j=1}^{J} \xi_{j} \mathcal{I}_{C M} \tilde{u}_{C R, j}\right)-\lambda_{J}+\left|\lambda_{J}-\tilde{\lambda}_{C R, J}\right|+\tilde{\lambda}_{C R, J}^{2} \kappa^{2} H^{2}+\|\mathbf{r}\|_{\mathbf{B}^{-1}} .
$$

Suppose that $\xi_{1}, \ldots, \xi_{J}$ denote some coefficients of a maximiser $\tilde{v}_{C}$ in the Rayleigh quotient of (5.4) $, \tilde{v}_{C}:=\sum_{j=1}^{J} \xi_{j} \mathcal{I}_{C M} \tilde{u}_{C R, j}$, and set $\tilde{v}_{C R}:=\sum_{j=1}^{J} \xi_{j} \tilde{u}_{C R, j}$. Here, the arguments of step two in the proof of Lemma 4.2 lead to

$$
R\left(\tilde{v}_{C}\right)-\lambda_{J} \lesssim\left\|u_{J}-\tilde{v}_{C R}\right\|_{N C}+\left\|\tilde{v}_{C R}-\tilde{v}_{C}\right\|_{N C}+\left(\left\|\tilde{v}_{C}\right\|-1\right)^{2} R\left(\tilde{v}_{C}\right) .
$$

Since $\tilde{u}_{C R, 1}, \ldots, \tilde{u}_{C R, J}$ is orthonormal and, without loss of generality, $\xi_{1}^{2}+\ldots+\xi_{J}^{2}=1$, it holds that $\left\|\tilde{v}_{C R}\right\|=1$ and

$$
\left(\left\|\tilde{v}_{C}\right\|-1\right)^{2} \leq\left\|\tilde{v}_{C R}-\tilde{v}_{C}\right\|^{2} .
$$


The discrete scaling argument of (4.4) implies

$$
\left\|\tilde{v}_{C R}-\tilde{v}_{C}\right\| \lesssim H\left\|\tilde{v}_{C R}-\tilde{v}_{C}\right\|_{N C} .
$$

The linearity of $\mathcal{I}_{C M}$ from Lemma 3.5, $\tilde{v}_{C R}-\tilde{v}_{C}=\tilde{v}_{C R}-\mathcal{I}_{C M} \tilde{v}_{C R}$, plus Lemma 3.4 show that

$$
\left\|\tilde{v}_{C R}-\tilde{v}_{C}\right\|_{N C} \lesssim\left\|u_{J}-\tilde{v}_{C R}\right\|_{N C}
$$

The arguments of steps three and four in the proof of Lemma 4.2 plus Lemma 4.3 and Theorem 4.4 conclude the proof of the efficiency.

Example 5.3. The criss-cross triangulation of Figure 1.1 leads to the matrices $\mathbf{A}=4 I_{4 \times 4}=\mathbf{B} / 24$ for the $4 \times 4$-dimensional identity matrix $I_{4 \times 4}$. Any vector $\mathbf{u}_{C R} \in \mathbb{R}^{4}$ is an eigenvector with $\mathbf{r} \equiv 0$ to the eigenvalue $\lambda_{C R}=24$ of multiplicity four. For $J=2$ one may choose the basis $\left(u_{C R, 1}, u_{C R, 2}\right)$ proportional to $(1,1,1,1)$ and $(1,1,1,0)$ that leads to the bounds $4.2594 \leq \lambda_{2} \leq 72$. Note that the exact second and third eigenvalues $\lambda_{2}=\lambda_{3}=5 \pi^{2}=49.348$ coincide. The condition $H<(\sqrt{3 / 2}-1) /(\kappa \pi \sqrt{5})$ is violated, but some elementary direct considerations with $u_{2}$ and $u_{3}$ on the continuous level and the positivity of $u_{1}$ imply that $\mathcal{I}_{N C} u_{1}$ and $\mathcal{I}_{N C} u_{2}$ are linearly independent. Therefore, the aforementioned eigenvalue bounds for $\lambda_{2}$ are guaranteed. The eigenvalue bounds are remarkable in that $J=2$ cuts a cluster of eigenvalues on the continuous level $\left(\lambda_{2}=\lambda_{3}\right)$ as well as on the discrete level $\left(\lambda_{C R, 1}=\ldots=\lambda_{C R, 4}\right)$.

Remark 5.4. Note that Lemma 5.2 provides an explicit bound for the global meshsize that leads to the separation condition in Theorem 5.1 but does not need any regularity assumption of the eigenfunctions. Elliptic regularity for some convex domain $\Omega$ Gri85, Theorem 4.3.1.4] shows

$$
\left\|D^{2} u_{J}\right\|=\left\|\Delta u_{J}\right\|=\lambda_{J}\left\|u_{J}\right\|=\lambda_{J} .
$$

Since (3.1), the Poincaré inequality on a triangle $T \in \mathcal{T}$ [LS10] reads

$$
\left\|\nabla\left(u_{J}-\mathcal{I}_{N C} u_{J}\right)\right\|_{L^{2}(T)}^{2} \leq h_{T}^{2} / j_{1,1}^{2}\left\|D^{2} u_{J}\right\|_{L^{2}(T)}^{2} .
$$

The square roots of the sum of all those inequalities reads

$$
\left\|u_{J}-\mathcal{I}_{N C} u_{J}\right\|_{N C} \leq H / j_{1,1}\left\|D^{2} u_{J}\right\|_{L^{2}(\Omega)} .
$$

This and Theorem 2.1 plus the aforementioned elliptic regularity estimate shows

$$
\left\|u_{J}-\mathcal{I}_{N C} u_{J}\right\| \leq \kappa H\left\|u_{J}-\mathcal{I}_{N C} u_{J}\right\|_{N C} \leq \kappa H^{2} / j_{1,1}\left\|D^{2} u_{J}\right\| \leq \kappa H^{2} \lambda_{J} / j_{1,1} .
$$

This leads to the improved separation condition

$$
H^{2}<j_{1,1}(\sqrt{1+1 / J}-1) /\left(\kappa \lambda_{J}\right)
$$

for higher eigenvalues on convex domains in Theorem [5.1. The reduced elliptic regularity allows a similar proof with rather unknown constants from $\left\|u_{J}\right\|_{H^{s}(\Omega)} \leq$ $C(s)\left\|\lambda_{J} u_{J}\right\|$.

\section{NumericAl EXPERIMENTS}

This section presents an adaptive algorithm and provides some numerical examples for the unit square, the L-shaped domain, and two isospectral domains. 

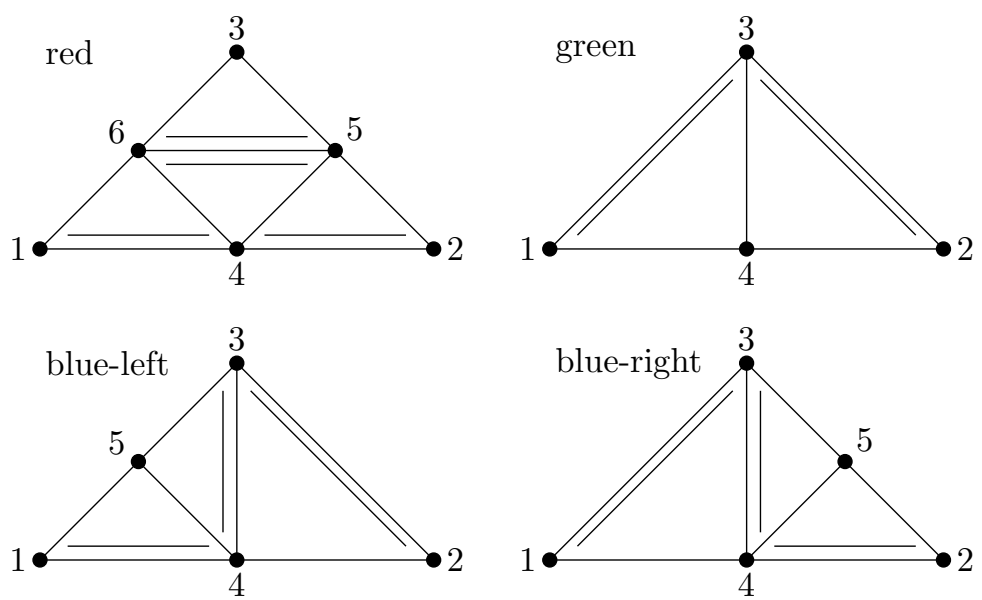

FiguRE 6.1. Refinement rules: sub-triangles with corresponding reference edges depicted with a second edge.

6.1. Adaptive finite element algorithm. The basic adaptive finite element method (AFEM) starts from an initial coarse triangulation $\mathcal{T}_{0}$ and generates a sequence of nested triangulations $\mathcal{T}_{0}, \mathcal{T}_{1}, \ldots$ with corresponding nonnested nonconforming spaces $\left(C R_{0}^{1}\left(\mathcal{T}_{\ell}\right)\right)_{\ell}$ in successive loops of the form

$$
\text { Solve } \longrightarrow \text { Estimate } \longrightarrow \text { Mark } \longrightarrow \text { Refine. }
$$

Input. $\mathcal{T}_{0}, 0<\theta \leq 1, \tau>0$.

Solve. Input: Approximation $\left(\tilde{\lambda}_{1, \ell}, \tilde{u}_{1, \ell}\right) \in \mathbb{R} \times C R_{0}^{1}\left(\mathcal{T}_{\ell}\right)$ on the triangulation $\mathcal{T}_{\ell}$.

\section{Repeat}

Run one iteration step of the preconditioned inverse iteration (PIN-

VIT) KN03] with one V-cycle multigrid iteration with Richardson smoother Bre99] as a preconditioner

until

$$
\left\|\mathbf{r}_{\ell}\right\|_{\mathbf{B}_{\ell}^{-1}} \leq \min \left\{\tilde{\lambda}_{1, \ell}, \tau\right\}, \mathbf{r}_{\ell}:=\mathbf{A}_{\ell} \tilde{\mathbf{u}}_{1, \ell}-\tilde{\lambda}_{1, \ell} \mathbf{B}_{\ell} \tilde{\mathbf{u}}_{1, \ell}
$$

and if $1>\kappa^{4}\left(\tilde{\lambda}_{1, \ell}-\left\|\mathbf{r}_{\ell}\right\|_{\mathbf{B}_{\ell}^{-1}}\right)^{2} H_{\ell}^{4}$ until $\eta_{2} \leq \max \left\{\eta_{1}, \eta_{3}\right\}$.

Estimate. The error estimate of Theorem 3.1 reads

$$
\left|\lambda_{1}-\tilde{\lambda}_{1, \ell}\right| \leq \eta_{1}+\eta_{2}+\eta_{3}
$$

with

$$
\begin{aligned}
\eta_{1} & :=\frac{\tilde{\lambda}_{1, \ell} \kappa^{2}\left(\tilde{\lambda}_{1, \ell}-\left\|\mathbf{r}_{\ell}\right\|_{\mathbf{B}_{\ell}^{-1}}\right) H_{\ell}^{2}}{1-\kappa^{4}\left(\tilde{\lambda}_{1, \ell}-\left\|\mathbf{r}_{\ell}\right\|_{\mathbf{B}_{\ell}^{-1}}\right)^{2} H_{\ell}^{4}}, \\
\eta_{2} & :=\frac{\left\|\mathbf{r}_{\ell}\right\|_{\mathbf{B}_{\ell}^{-1}}}{1+\kappa^{2}\left(\tilde{\lambda}_{1, \ell}-\left\|\mathbf{r}_{\ell}\right\|_{\mathbf{B}_{\ell}^{-1}}\right) H_{\ell}^{2}}, \\
\eta_{3} & :=R\left(\mathcal{I}_{C M} \tilde{u}_{1, \ell}\right)-\frac{\tilde{\lambda}_{1, \ell}}{1-\kappa^{4}\left(\tilde{\lambda}_{1, \ell}-\left\|\mathbf{r}_{\ell}\right\|_{\mathbf{B}_{\ell}^{-1}}\right)^{2} H_{\ell}^{4}}
\end{aligned}
$$


TABLE 6.1. Spectral gap for the smallest eigenvalue of the unit square for different meshes with $N=\left|\mathcal{E}_{\ell}(\Omega)\right|$ degrees of freedom.

\begin{tabular}{|c|c|c|c|c|}
\hline$N$ & 16 & 56 & 208 & 800 \\
\hline$\lambda_{2, \ell}-\lambda_{1, \ell}$ & 12.0964 & 25.4691 & 28.5894 & 29.3549 \\
\hline \hline$N$ & 3136 & 12416 & 49408 & 197120 \\
\hline$\lambda_{2, \ell}-\lambda_{1, \ell}$ & 29.5454 & 29.5930 & 29.6048 & 29.6078 \\
\hline
\end{tabular}

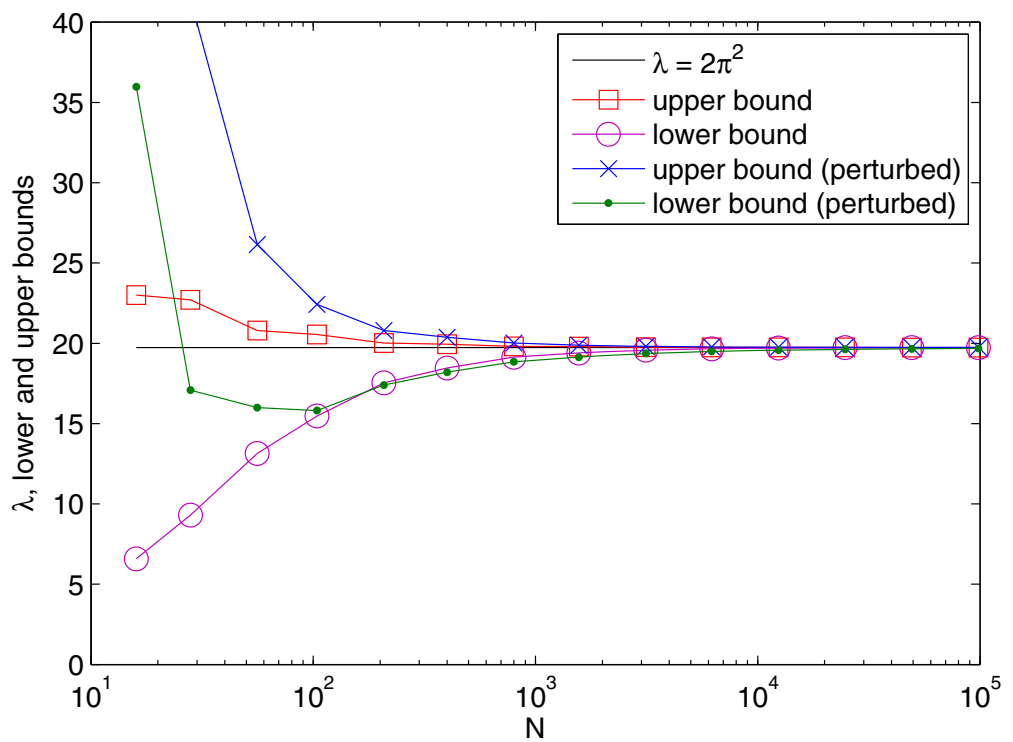

Figure 6.2. Computed and randomly perturbed upper and lower bounds on the smallest eigenvalue of the unit square

Mark. The mesh-refinement selects a set of edges $\mathcal{M}_{\ell} \subseteq \mathcal{E}_{\ell}$ with the goal to balance the contributions $\eta_{1}+\eta_{2}+\eta_{3}$ as follows:

(a) If $1 \leq \kappa^{4}\left(\tilde{\lambda}_{1, \ell}-\left\|\mathbf{r}_{\ell}\right\|_{\mathbf{B}_{\ell}^{-1}}\right)^{2} H_{\ell}^{4}$ or $\eta_{1}>\max \left\{\eta_{2}, \eta_{3}\right\}$ then $\mathcal{M}_{\ell}:=\bigcup\left\{E \in \mathcal{E}_{\ell}\right.$ : $\left.|E|=H_{\ell}\right\}$.

(b) Else if $\eta_{3} \geq \max \left\{\eta_{1}, \eta_{2}\right\}$, then the set of marked edges $\mathcal{M}_{\ell} \subseteq \mathcal{E}_{\ell}$ is of minimal cardinality that fulfills the bulk criterion Dör96]:

$$
\theta \sum_{E \in \mathcal{E}_{\ell}} \eta_{\ell}^{2}(E) \leq \sum_{E \in \mathcal{M}_{\ell}} \eta_{\ell}^{2}(E) \quad \text { for } \quad \eta_{\ell}^{2}(E):=h_{E}\left\|\left[\partial \tilde{u}_{1, \ell} / \partial s\right]\right\|_{L^{2}(E)}^{2} .
$$

Refine. Given the set $\mathcal{M}_{\ell} \subseteq \mathcal{E}_{\ell}$ of marked edges, the refinement $\mathcal{T}_{\ell+1}$ is computed as a minimal regular triangulation such that $\mathcal{M}_{\ell} \subseteq \mathcal{E}_{\ell} \backslash \mathcal{E}_{\ell+1}$ and each triangle is refined by one of the rules from Figure 6.1.

6.2. Unit square. Consider the model problem (1.1) on the unit square $\Omega=(0,1)^{2}$ with the smallest eigenvalue $\lambda_{1}=2 \pi^{2}$. 


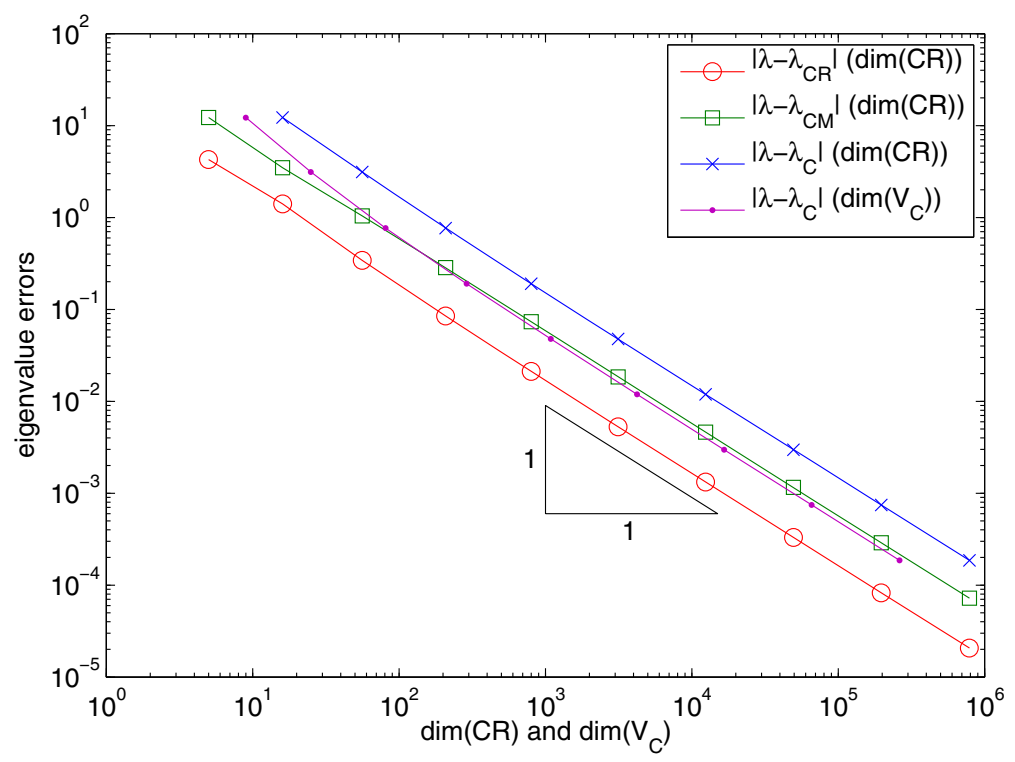

Figure 6.3. Convergence history for the unit square for different eigenvalue errors

The first experiment in Table 6.1 investigates the critical algebraic condition on the spectral gap $\lambda_{2, \ell}-\lambda_{1, \ell}$. The results are computed for a sequence of red-refined meshes and the ARPACK LSY98 solver (implemented in the Matlab function 'eigs') with tolerance up to machine precision. The spectral gap is relatively large even for coarse meshes and motivates the choice $\tau=1$.

Figure 6.2 verifies that the lower and upper bounds of Theorem 3.1 are empirically lower and upper eigenvalue bounds and presents some perturbed bounds as well. The perturbed bounds are obtained from a perturbed eigenvector

$$
\tilde{\mathbf{u}}_{1, \ell}=\mathbf{u}_{1, \ell}+\operatorname{rand}(0,1) /\left(\operatorname{dim}\left(C R_{0}^{1}\left(\mathcal{T}_{\ell}\right)\right) \lambda_{1, \ell}\right),
$$

where $\mathbf{u}_{1, \ell}$ is computed with ARPACK up to machine precision. The perturbed eigenvalue is the Rayleigh quotient of the perturbed eigenvector. Note that the numerical results show that for the first mesh the perturbation is too large such that a different eigenvalue is approximated and the lower bound does not hold.

Figure 6.3 compares the accuracy of the nonconforming and the conforming FEMs on uniform red-refined meshes. The first observation is that the nonconforming eigenvalue error $\left|\lambda_{1}-\lambda_{C R, 1}\right|$ is smaller than the conforming eigenvalue error $\left|\lambda_{1}-\lambda_{C, 1}\right|$ displayed versus its degrees of freedoms $N:=\operatorname{dim}\left(V_{C}\right)$. The comparison of the conforming eigenvalue error $\left|\lambda_{1}-\lambda_{C, 1}\right|$ and the error for the postprocessing $\lambda_{C M, 1}:=R\left(\mathcal{I}_{C M} u_{C R, 1}\right),\left|\lambda_{1}-\lambda_{C M, 1}\right|$, both plotted versus $N=\operatorname{dim}\left(C R_{0}^{1}(\mathcal{T})\right)$, shows that the proposed interpolation on the red-refined mesh leads to better upper bounds than a conforming approximation on the coarse mesh.

6.3. L-shaped domain. Consider the model problem (1.1) on the L-shaped domain $\Omega=(-1,1)^{2} \backslash([0,1] \times[-1,0])$ with $\lambda_{1}=9.6397238440219$ TB06].

Figure 6.4 compares the eigenvalue error for the mean value $\mu$ of the upper and lower eigenvalue bounds in Theorem 3.1 to its upper bound $\eta / 2$. Uniform 


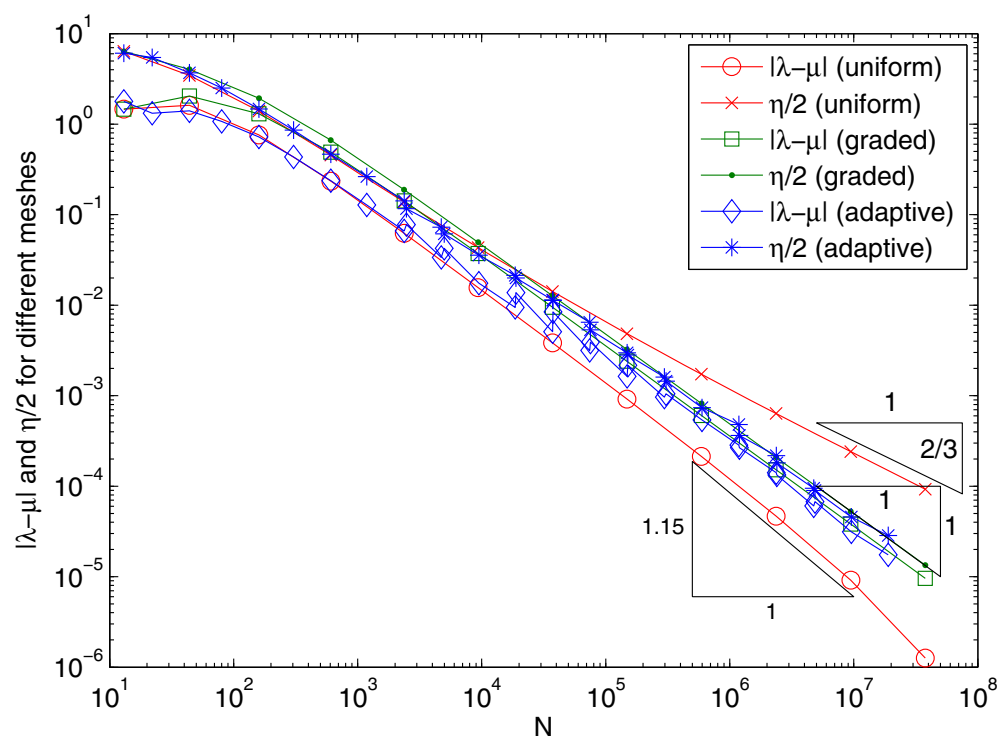

FiguRE 6.4. Convergence history for the L-shaped domain

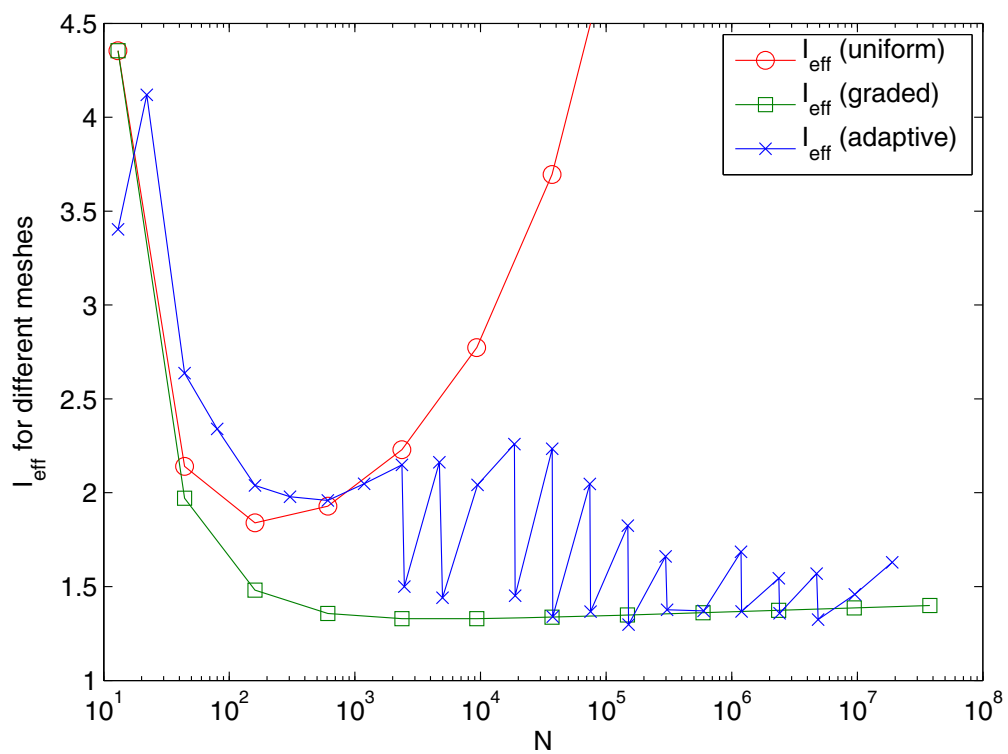

FiguRE 6.5. Efficiency indices for the L-shaped domain

red-refined meshes with ARPACK result in sub-optimal convergence of the estimator $\eta / 2$ as expected for the singular eigenfunction but lead to a surprising superconvergence of the error $\left|\lambda_{1}-\mu\right|$. The surprising super-convergence of $\left|\lambda_{1}-\mu\right|$ might result from some super-convergence phenomena on this highly structured grid; cf. WZ09 for super-convergence phenomena of eigenvalues. For graded meshes with ARPACK the empirical convergence rate is optimal and for the proposed adaptive algorithm it is asymptotically optimal. The eigenvalue error of the adaptive algorithm is not monotone which results from the fact that the algorithm starts with 

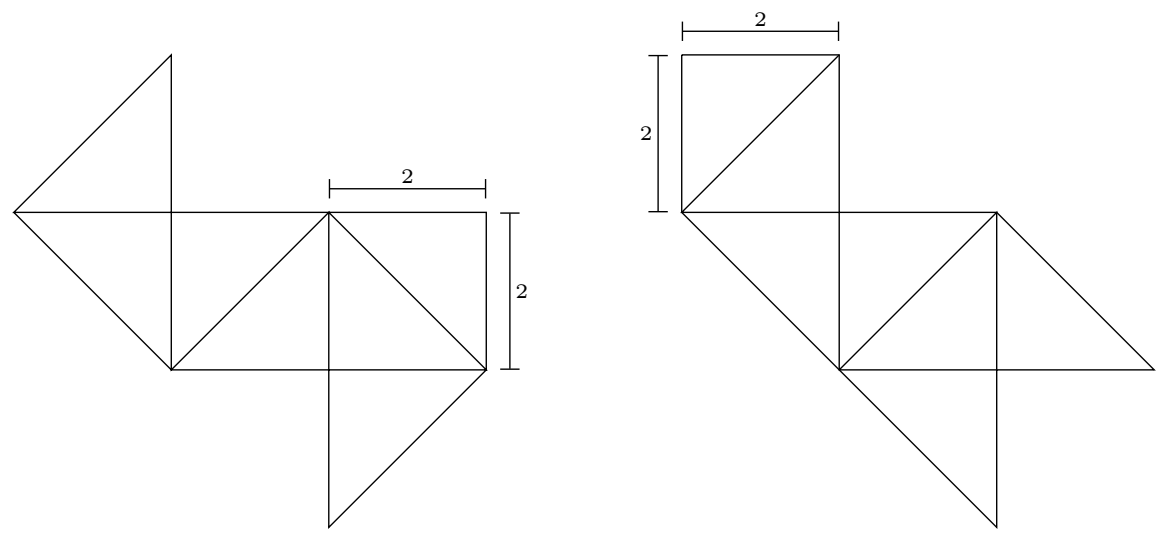

Figure 6.6. Two isospectral domains

uniform refinements at the beginning and therefore the error matches the superconvergent error. Afterwards one step of uniform refinement is followed by one step of adaptive refinement that destroys the mesh-symmetry and therefore the superconvergence. As a result the error gets closer and closer to the quasi-optimal error for graded meshes. In contrast to that the error bound $\eta / 2$ is monotonically decreasing. This illustrates the mixed adaptive strategy with respect to the algebraic eigenvalue error, the global mesh-size, and the approximation error and provides numerical evidence for the superiority of adaptive mesh-refinement.

Figure 6.5 displays the efficiency indices $I_{\text {eff }}:=(\eta / 2) /\left|\lambda_{1}-\mu\right|$. Clearly, for uniform meshes one observes the mentioned efficiency gap. The values for graded and adaptive meshes are between 1 and 2 and tend to 1.4. Since $\eta / 2$ is a guaranteed upper bound, all values are greater or equal to one.

6.4. Isospectral domains. Consider the model problem (1.1) on the two isospectral domains of Figure 6.6 with the approximation of the 50-th eigenvalue $\lambda_{50}=$ 54.187936 [TB06]. For the numerical experiments, both domains are triangulated similarly with the same number of triangles. The experiments show for uniform red-refinements and ARPACK that both domains lead to the same eigenvalue approximations up to machine precision. Table 6.2 verifies empirically the theoretical upper and lower bounds of Theorem 5.1 and shows that also the computed upper and lower bounds are equal up to machine precision for both domains. An interesting observation on the maximising Rayleigh quotient in Theorem 5.1 is that the maximum of $R\left(\xi_{1} \mathcal{I}_{C M} \tilde{u}_{C R, 1}+\ldots+\xi_{50} \mathcal{I}_{C M} \tilde{u}_{C R, 50}\right)$ is obtained for $\xi_{1}=\ldots=\xi_{45}=0$ and $\xi_{50}=1$ in all displayed numerical experiments. The separation condition of Theorem 5.1 leads in this example with $J=50$ to $H<0.007$ which is satisfied for the triangulations in the last and second last entry of Table 6.2. Remark 5.4 illustrates that this condition is coarse but explicit constants for the nonconvex domain at hand require more insight which is compensated by this strong separation condition in this paper. 
TABLE 6.2. Bounds for $\lambda_{50}=54.187936$ for the isospectral domains of Figure 6.6

\begin{tabular}{|c|c|c|}
\hline \multirow[b]{2}{*}{$\mathrm{N}$} & \multicolumn{2}{|c|}{ lower bounds } \\
\hline & left domain & right domain \\
\hline 186 & 8.484029241600799 & 8.484029241600801 \\
\hline 708 & 22.079541883464980 & 22.079541883464987 \\
\hline 2760 & 40.139305042643208 & 40.139305042643237 \\
\hline 10896 & 49.823736249152233 & 49.823736249152240 \\
\hline 43296 & 53.022275017108896 & 53.022275017108903 \\
\hline 172608 & 53.889870459421545 & 53.889870459421537 \\
\hline 689280 & 54.112360562895724 & 54.112360562895560 \\
\hline 2754816 & 54.168723796821510 & 54.168723796821538 \\
\hline 11014656 & 54.183012990240513 & 54.183012990240186 \\
\hline & \multicolumn{2}{|c|}{ upper bounds } \\
\hline $\mathrm{N}$ & left domain & right domain \\
\hline 186 & 114.2653311991490 & 114.2653311991488 \\
\hline 708 & $64.39713286238 \mathbf{6 2 5 8}$ & 64.397132862387565 \\
\hline 2760 & 56.619351329573185 & 56.619351329573249 \\
\hline 10896 & 54.818424684560334 & 54.818424684560306 \\
\hline 43296 & 54.352753736838082 & 54.352753736838132 \\
\hline 172608 & 54.231273697990432 & 54.231273697990602 \\
\hline 689280 & 54.199573365120656 & 54.199573365121147 \\
\hline 2754816 & 54.191162363149061 & 54.191162363147861 \\
\hline 11014656 & 54.188868310930701 & 54.188868310929948 \\
\hline
\end{tabular}

\section{ACKNOWLEDGMENTS}

The authors would like to thank the anonymous referees for their valuable comments and suggestions.

\section{REFERENCES}

[AD04] María G. Armentano and Ricardo G. Durán, Asymptotic lower bounds for eigenvalues by nonconforming finite element methods, Electron. Trans. Numer. Anal. 17 (2004), 93-101 (electronic). MR2040799 (2005d:65198)

[BO91] I. Babuška and J. Osborn, Eigenvalue problems, Handbook of numerical analysis, Vol. II, Handb. Numer. Anal., II, North-Holland, Amsterdam, 1991, pp. 641-787. MR 1115240

[Bre99] Susanne C. Brenner, Convergence of nonconforming multigrid methods without full elliptic regularity, Math. Comp. 68 (1999), no. 225, 25-53, DOI 10.1090/S0025-571899-01035-2. MR1620215 (99c:65229)

[CELH12] C. Carstensen, M. Eigel, R. H. W. Hoppe, and C. Löbhard, A review of unified a posteriori finite element error control, Numer. Math. Theory Methods Appl. 5 (2012), no. 4, 509-558, DOI 10.4208/nmtma.2011.m1032. MR.2968740

[CG11] Carsten Carstensen and Joscha Gedicke, An oscillation-free adaptive FEM for symmetric eigenvalue problems, Numer. Math. 118 (2011), no. 3, 401-427, DOI 10.1007/s00211-011-0367-2. MR2810801 (2012h:65262)

[CG12a] C. Carstensen and D. Gallistl, Guaranteed lower eigenvalue bounds for the biharmonic equation, Numer. Math. 126 (2014), no. 1, 33-51. MR3149071 
[CG12b] Carsten Carstensen and Joscha Gedicke, An adaptive finite element eigenvalue solver of asymptotic quasi-optimal computational complexity, SIAM J. Numer. Anal. 50 (2012), no. 3, 1029-1057, DOI 10.1137/090769430. MR2970733

[CGR12] Carsten Carstensen, Joscha Gedicke, and Donsub Rim, Explicit error estimates for Courant, Crouzeix-Raviart and Raviart-Thomas finite element methods, J. Comput. Math. 30 (2012), no. 4, 337-353, DOI 10.4208/jcm.1108-m3677. MR.2965987

[CM13] C. Carstensen and C. Merdon, Computational survey on a posteriori error estimators for nonconforming finite element methods for the Poisson problem, J. Comput. Appl. Math. 249 (2013), 74-94, DOI 10.1016/j.cam.2012.12.021. MR.3037808

[DDP12] E. A. Dari, R. G. Durán, and C. Padra, A posteriori error estimates for nonconforming approximation of eigenvalue problems, Appl. Numer. Math. 62 (2012), no. 5, 580-591, DOI 10.1016/j.apnum.2012.01.005. MR2899264

[DDPV96] E. Dari, R. Duran, C. Padra, and V. Vampa, A posteriori error estimators for nonconforming finite element methods (English, with English and French summaries), RAIRO Modél. Math. Anal. Numér. 30 (1996), no. 4, 385-400. MR1399496 (97f:65066)

[Dör96] Willy Dörfler, A convergent adaptive algorithm for Poisson's equation, SIAM J. Numer. Anal. 33 (1996), no. 3, 1106-1124, DOI 10.1137/0733054. MR.1393904 (97e:65139)

[DPR03] Ricardo G. Durán, Claudio Padra, and Rodolfo Rodríguez, A posteriori error estimates for the finite element approximation of eigenvalue problems, Math. Models Methods Appl. Sci. 13 (2003), no. 8, 1219-1229, DOI 10.1142/S0218202503002878. MR.1998821 (2004f:65173)

[DRSZ08] Wolfgang Dahmen, Thorsten Rohwedder, Reinhold Schneider, and Andreas Zeiser, Adaptive eigenvalue computation: complexity estimates, Numer. Math. 110 (2008), no. 3, 277-312, DOI 10.1007/s00211-008-0159-5. MR2430982(2009e:65162)

[DXZ08] Xiaoying Dai, Jinchao Xu, and Aihui Zhou, Convergence and optimal complexity of adaptive finite element eigenvalue computations, Numer. Math. 110 (2008), no. 3, 313-355, DOI 10.1007/s00211-008-0169-3. MR2430983(2009h:65166)

[Eva00] Lawrence C. Evans, Partial differential equations, Graduate Studies in Mathematics, vol. 19, American Mathematical Society, Providence, RI, 1998. MR.1625845 (99e:35001)

[For55] George E. Forsythe, Asymptotic lower bounds for the fundamental frequency of convex membranes, Pacific J. Math. 5 (1955), 691-702. MR0073048 (17,373a)

[GG09] S. Giani and I. G. Graham, A convergent adaptive method for elliptic eigenvalue problems, SIAM J. Numer. Anal. 47 (2009), no. 2, 1067-1091, DOI 10.1137/070697264. MR2485445(2010b:65244)

[GMZ09] Eduardo M. Garau, Pedro Morin, and Carlos Zuppa, Convergence of adaptive finite element methods for eigenvalue problems, Math. Models Methods Appl. Sci. 19 (2009), no. 5, 721-747, DOI 10.1142/S0218202509003590. MR2531037(2010h:65212)

[Gri85] P. Grisvard, Elliptic problems in nonsmooth domains, Monographs and Studies in Mathematics, vol. 24, Pitman (Advanced Publishing Program), Boston, MA, 1985. $\operatorname{MR} 775683(86 \mathrm{~m}: 35044)$

[HHL11] J. Hu, Y. Huang, and Q. Lin, The lower bounds for eigenvalues of elliptic operators by nonconforming finite element methods, preprint, arXiv: 1112.1145v1, 2011.

[KN03] Andrew V. Knyazev and Klaus Neymeyr, A geometric theory for preconditioned inverse iteration. III. A short and sharp convergence estimate for generalized eigenvalue problems, Linear Algebra Appl. 358 (2003), 95-114, DOI 10.1016/S00243795(01)00461-X. Special issue on accurate solution of eigenvalue problems (Hagen, 2000). MR.1942725 (2004c:65040)

[LS10] R. S. Laugesen and B. A. Siudeja, Minimizing Neumann fundamental tones of triangles: an optimal Poincaré inequality, J. Differential Equations 249 (2010), no. 1, 118-135, DOI 10.1016/j.jde.2010.02.020. MR2644129(2011f:35238)

[LSY98] R. B. Lehoucq, D. C. Sorensen, and C. Yang, ARPACK users' guide, Software, Environments, and Tools, vol. 6, Society for Industrial and Applied Mathematics (SIAM), Philadelphia, PA, 1998. Solution of large-scale eigenvalue problems with implicitly restarted Arnoldi methods. MR.1621681 
[Par98] Beresford N. Parlett, The symmetric eigenvalue problem, Classics in Applied Mathematics, vol. 20, Society for Industrial and Applied Mathematics (SIAM), Philadelphia, PA, 1998. Corrected reprint of the 1980 original. MR1490034 (99c:65072)

[SF73] Gilbert Strang and George J. Fix, An analysis of the finite element method, PrenticeHall Inc., Englewood Cliffs, N. J., 1973. Prentice-Hall Series in Automatic Computation. MR0443377 (56 \#1747)

[TB06] Lloyd N. Trefethen and Timo Betcke, Computed eigenmodes of planar regions, Recent advances in differential equations and mathematical physics, Contemp. Math., vol. 412, Amer. Math. Soc., Providence, RI, 2006, pp. 297-314, DOI 10.1090/conm/412/07783. MR2259116(2008a:35042)

[Wei56] H. F. Weinberger, Upper and lower bounds for eigenvalues by finite difference methods, Comm. Pure Appl. Math. 9 (1956), 613-623. MR0084185 (18,826a)

[WZ09] Haijun Wu and Zhimin Zhang, Enhancing eigenvalue approximation by gradient recovery on adaptive meshes, IMA J. Numer. Anal. 29 (2009), no. 4, 1008-1022, DOI 10.1093/imanum/drn050. MR2557054(2010k:65249)

Institut für Mathematik, Humboldt-Universität Zu Berlin, Unter den Linden 6, 10099 Berlin, Germany - And - Department of Computational Science and Engineering, YonSEI UNiversity, 120-749 SeOul, Korea.

E-mail address: cc@mathematik.hu-berlin.de

Institut für Mathematik, Humboldt-Universität Zu Berlin, Unter den Linden 6, 10099 Berlin, Germany

Current address: Department of Mathematics and Center for Computation and Technology, Louisiana State University, Baton Rouge, Louisiana 70803

E-mail address: jgedicke@math.1su.edu 